\title{
Ultralight scalars in leptonic observables
}

\author{
Pablo Escribano $^{a}$ and Avelino Vicente ${ }^{a, b}$ \\ ${ }^{a}$ Instituto de Física Corpuscular, CSIC-Universitat de València, \\ C/ Catedrático José Beltrán 2, E-46980 Paterna, Valencia, Spain \\ ${ }^{b}$ Departament de Fúsica Teòrica, Universitat de València, \\ C/ Dr. Moliner 50, E-46100 Burjassot, Valencia, Spain \\ E-mail: pablo.escribano@ific.uv.es, avelino.vicente@ific.uv.es
}

\begin{abstract}
Many new physics scenarios contain ultralight scalars, states which are either exactly massless or much lighter than any other massive particle in the model. Axions and majorons constitute well-motivated examples of this type of particle. In this work, we explore the phenomenology of these states in low-energy leptonic observables. After adopting a model independent approach that includes both scalar and pseudoscalar interactions, we briefly discuss the current limits on the diagonal couplings to charged leptons and consider processes in which the ultralight scalar $\phi$ is directly produced, such as $\mu \rightarrow e \phi$, or acts as a mediator, as in $\tau \rightarrow \mu \mu \mu$. Contributions to the charged leptons magnetic and electric moments are studied as well.
\end{abstract}

Keywords: Beyond Standard Model, CP violation, Neutrino Physics

ArXiv EPrint: 2008.01099 


\section{Contents}

1 Introduction 1

2 Effective Lagrangian $\quad 3$

3 Bounds on lepton flavor conserving couplings 4

3.1 Stellar cooling 4

$\begin{array}{ll}3.2 & \text { 1-loop coupling to photons }\end{array}$

4 Leptonic observables $\quad 8$

$4.1 \ell_{\alpha} \rightarrow \ell_{\beta} \phi$

$4.2 \ell_{\alpha} \rightarrow \ell_{\beta} \gamma \phi \quad 9$

$4.3 \ell_{\alpha} \rightarrow \ell_{\beta} \gamma \quad 10$

$4.4 \ell_{\alpha}^{-} \rightarrow \ell_{\beta}^{-} \ell_{\beta}^{-} \ell_{\beta}^{+} \quad 12$

$4.5 \ell_{\alpha}^{-} \rightarrow \ell_{\beta}^{-} \ell_{\gamma}^{-} \ell_{\gamma}^{+} \quad 13$

$4.6 \ell_{\alpha}^{-} \rightarrow \ell_{\beta}^{+} \ell_{\gamma}^{-} \ell_{\gamma}^{-} \quad 14$

$\begin{array}{lll}4.7 & \text { Lepton magnetic and electric dipole moments } & 15\end{array}$

5 Phenomenological discussion $\quad 17$

$\begin{array}{lll}5.1 & \text { Searches for } \ell_{\alpha} \rightarrow \ell_{\beta} \phi & 17\end{array}$

$5.2 \ell_{\alpha} \rightarrow \ell_{\beta} \gamma \phi$ at the MEG experiment 18

$5.3 \ell_{\alpha} \rightarrow \ell_{\beta} \gamma$ vs. $\ell_{\alpha} \rightarrow \ell_{\beta} \ell_{\beta} \ell_{\beta}$

5.4 Lepton magnetic and electric dipole moments 24

$\begin{array}{llr}6 & \text { Conclusions } & 27\end{array}$

$\begin{array}{ll}\text { A Parametrization in terms of derivative interactions } & 28\end{array}$

\section{Introduction}

Lepton flavor physics is about to live a golden age. Several state-of-the-art experiments recently started taking data and a few more are about to begin [1]. These include new searches for lepton flavor violating (LFV) processes, forbidden in the Standard Model (SM), as well as more precise measurements of lepton flavor conserving observables, such as charged lepton anomalous magnetic moments. The search for LFV in processes involving charged leptons is strongly motivated by the observation of LFV in the neutral sector (in the form of neutrino flavor oscillations). In what concerns muon observables, the search for the radiative LFV decay $\mu \rightarrow e \gamma$ is going to be led by the second phase of the MEG experiment, MEG-II [2,3], while the long-awaited Mu3e experiment will aim at an impressive sensitivity to branching ratios for the 3 -body decay $\mu \rightarrow$ eee as low as $10^{-16}[3,4]$. A plethora of 
promising experiments looking for neutrinoless $\mu-e$ conversion in nuclei is also planned. Flavor factories and experiments aiming at a broad spectrum of flavor observables, such as Belle II and LHCb, will also contribute to this era of lepton flavor, mainly due to their high sensitivities in the measurement of tau lepton observables [5,6]. On the flavor conserving side, improved measurements of the muon anomalous magnetic moment are expected at the Muon g-2 experiment [7], hopefully shedding light on a well-known longstanding experimental anomaly.

With such an exciting experimental perspective in the coming years, it is natural to ask what type of new physics can be probed. In this work we will concentrate on ultralight scalars that couple to charged leptons and study their impact on leptonic observables. In this context, we will use the term ultralight scalar to refer to a generic scalar $\phi$ that is much lighter than the electron, $m_{\phi} \ll m_{e}$, and can therefore be produced on-shell in charged lepton decays. In practice, this also means that $\phi$ can be assumed to be approximately massless in all considered physical processes. We will take a model independent approach and neglect $m_{\phi}$ in our analytical calculations. Actually, this is not an approximation if $\phi$ is exactly massless, the case for a Goldstone boson whose mass is protected by a (spontaneously broken) global continuous symmetry.

There are many well-known examples of such ultralight scalars. If the apparent absence of $\mathrm{CP}$ violation in the strong interactions is explained by means of the Peccei-Quinn mechanism [8], a new pseudoscalar state must exist: the axion [9, 10]. Although its mass is not predicted and can vary over a wide range of scales [11], a large fraction of the parameter space (corresponding to large axion decay constants) leads to an ultralight axion. Interestingly, such low mass axion would be of interest as a possible component of the dark matter of the Universe [12-14]. Axion-like particles, or ALPs, generalize this type of scenario by making the mass and decay constant two independent parameters. This allows for a larger parameter space, again including a substantial portion with very low ALP masses. The solution to the strong CP problem could also be intimately related to the flavor problem of the SM $[15,16]$. This naturally leads to a flavored axion [17-20], although an axion with flavor-blind interactions is also possible [21]. Another popular ultralight scalar is the majoron, the Goldstone boson associated to the breaking of global lepton number [22-25]. While this state can gain a small mass by various mechanisms, and then be a possible dark matter candidate [26, 27], it is expected to be exactly massless in the absence of explicit breaking of lepton number. Another possible ultralight scalar is the familon, the Goldstone boson of spontaneously broken global family symmetry. Finally, the Universe could also be filled with ultralight scalars in the form of fuzzy cold dark matter [28].

While many of the previously discussed examples are pseudoscalar states, the ultralight scalar $\phi$ can also have pure scalar couplings. This would be the case for a massless Goldstone boson if the associated global symmetry is non-chiral. Therefore, restricting the phenomenological exploration to just pseudoscalars would miss a relatively large number of well-motivated scenarios. This has actually been the case in many recent works [29-39], which were mainly interested in the phenomenology of flavored axions (or ALPs) and majorons [40]. 
Motivated by the principle of generality, we will consider a generic scenario where the $\mathrm{CP}$ nature of $\phi$ is not determined and explore several leptonic observables of interest. These include processes in which $\phi$ is produced in the final state, such as $\ell_{\alpha} \rightarrow \ell_{\beta} \phi$ or $\ell_{\alpha} \rightarrow \ell_{\beta} \phi \gamma$. In this case, we will generalize previous results in the literature, typically obtained for pure pseudoscalars or for the case of a massive $\phi$. We will also study processes in which $\phi$ is not produced, but acts as a mediator. A prime example of this category is $\ell_{\alpha}^{-} \rightarrow \ell_{\beta}^{-} \ell_{\beta}^{-} \ell_{\beta}^{+}$. To the best of our knowledge, the mediation of this process by an ultralight axion has only been previously considered in [29]. We will extend the study to more general scalar states and provide detailed analytical expressions for the decay width of the process. The analogous $\ell_{\alpha}^{-} \rightarrow \ell_{\beta}^{-} \ell_{\gamma}^{-} \ell_{\gamma}^{+}$and $\ell_{\alpha}^{-} \rightarrow \ell_{\beta}^{+} \ell_{\gamma}^{-} \ell_{\gamma}^{-}$decays will also be studied, in this case for the first time here. Charged lepton anomalous magnetic moments constitute other interesting examples of observables induced by the ultralight $\phi$.

The rest of the manuscript is organized as follows. We introduce our general setup, as well as our notation and conventions, in section 2 . In section 3 we discuss the current bounds on the lepton flavor conserving couplings of the scalar $\phi$. These are often constrained by studing their impact on astrophysical processes, but also receive indirect bounds due to their contribution to the 1-loop coupling of $\phi$ to photons, as we will show. In section 4 we discuss the impact of $\phi$ on several leptonic observables and derive analytical expressions for them. Phenomenological implications are considered in section 5. We summarize our findings and conclude in section 6. Finally, a pedagogical discussion on an alternative parametrization of the $\phi$ Lagrangian in terms of derivative interactions is provided in appendix A.

\section{Effective Lagrangian}

We are interested in charged leptons processes taking place at low energies in the presence of the ultralight real scalar $\phi$. For practical purposes, we will consider $\phi$ to be exactly massless, but our results are equally valid for a massive $\phi$, as long as $m_{\phi} \ll m_{e}$ holds. The interaction of the scalar $\phi$ with a pair of charged leptons $\ell_{\alpha}$ and $\ell_{\beta}$, with $\alpha, \beta=e, \mu, \tau$, can be generally parametrized by

$$
\mathcal{L}_{\ell \ell \phi}=\phi \bar{\ell}_{\beta}\left(S_{L}^{\beta \alpha} P_{L}+S_{R}^{\beta \alpha} P_{R}\right) \ell_{\alpha}+\text { h.c. },
$$

where $P_{L, R}=\frac{1}{2}\left(1 \mp \gamma_{5}\right)$ are the usual chiral projectors. No sum over the $\alpha$ and $\beta$ charged lepton flavor indices is performed. $S_{L}$ and $S_{R}$ are dimensionless coefficients and we consider all possible flavor combinations: $\beta \alpha=\{e e, \mu \mu, \tau \tau, e \mu, e \tau, \mu \tau\}$. Eq. (2.1) describes the most general effective interaction between the ultralight scalar $\phi$ and a pair of charged leptons. In particular, we note that eq. (2.1) includes both scalar and pseudoscalar interactions as well as flavor violating (charged lepton fields with $\alpha \neq \beta$ ) and flavor conserving (charged lepton fields with $\alpha=\beta$ ) interactions. An alternative parametrization for this Lagrangian based on the introduction of derivative interactions, applicable to the case of pseudoscalar interactions only, is discussed in appendix A. 
Some of the LFV observables considered below receive contributions from the usual dipole and 4-fermion operators. Therefore, our full effective Lagrangian is given by

$$
\mathcal{L}=\mathcal{L}_{\ell \ell \phi}+\mathcal{L}_{\ell \ell \gamma}+\mathcal{L}_{4 \ell}
$$

with

$$
\begin{aligned}
\mathcal{L}_{\ell \ell \gamma} & =\frac{e m_{\alpha}}{2} \bar{\ell}_{\beta} \sigma^{\mu \nu}\left[\left(K_{2}^{L}\right)^{\beta \alpha} P_{L}+\left(K_{2}^{R}\right)^{\beta \alpha} P_{R}\right] \ell_{\alpha} F_{\mu \nu}+\text { h.c. }, \\
\mathcal{L}_{4 \ell} & =\sum_{\substack{I=S, V, T \\
X, Y=L, R}}\left(A_{X Y}^{I}\right)^{\beta \alpha \delta \gamma} \bar{\ell}_{\beta} \Gamma_{I} P_{X} \ell_{\alpha} \bar{\ell}_{\delta} \Gamma_{I} P_{Y} \ell_{\gamma}+\text { h.c. },
\end{aligned}
$$

where $F_{\mu \nu}=\partial_{\mu} A_{\nu}-\partial_{\nu} A_{\mu}$ is the electromagnetic field strength tensor, with $A_{\mu}$ the photon field, and we have defined $\Gamma_{S}=1, \Gamma_{V}=\gamma_{\mu}$ and $\Gamma_{T}=\sigma_{\mu \nu}$. No sum over the $\alpha, \beta, \gamma$ and $\delta$ charged lepton flavor indices is performed in eqs. (2.3) and (2.4). The coefficients $K_{2}^{X}$ and $A_{X Y}^{I}$, with $I=S, V, T$ and $X, Y=L, R$, have dimensions of mass ${ }^{-2}$. We assume $m_{\alpha}>m_{\beta}$ and therefore normalize the Lagrangian in eq. (2.3) by including the mass of the heaviest charged lepton in the process of interest. Eq. (2.3) contains the usual photonic dipole operators, which contribute to $\ell_{\alpha} \rightarrow \ell_{\beta} \gamma$ and lead to

$$
\Gamma\left(\ell_{\alpha} \rightarrow \ell_{\beta} \gamma\right)=\frac{e^{2} m_{\alpha}^{5}}{16 \pi}\left[\left|\left(K_{2}^{L}\right)^{\beta \alpha}\right|^{2}+\left|\left(K_{2}^{R}\right)^{\beta \alpha}\right|^{2}\right],
$$

while eq. (2.4) contains 4-lepton operators. In summary, the effective Lagrangian in eq. (2.2) corresponds to the one in [41], extended to include the new operators with the scalar $\phi$ introduced in eq. (2.1).

In the following, we will disregard $\phi$ interactions with quarks and concentrate on purely leptonic observables, such as the LFV decays $\ell_{\alpha} \rightarrow \ell_{\beta} \phi$ or $\ell_{\alpha} \rightarrow \ell_{\beta} \ell_{\beta} \ell_{\beta}$, and the electron and muon anomalous magnetic and electric dipole moments. Even though $\phi$ couplings to quarks are possible, and indeed present in specific realizations of our general scenario, the prime example being the QCD axion, they introduce a large model dependence. We also note that leptophilic ultralight scalars, such as the majoron, are also well-motivated possibilities that naturally appear in models with spontaneous violation of global lepton number.

\section{Bounds on lepton flavor conserving couplings}

Let us comment on the current experimental contraints on the lepton flavor conserving couplings of the scalar $\phi$. We will start discussing the stellar cooling mechanism. Since this subject has been extensively studied in the literature, and we do not want to delve further into the topic, only a brief outline will be presented. Then we will discuss another source of constraints, the 1-loop coupling between $\phi$ and a pair of photons.

\subsection{Stellar cooling}

The production of $\phi$ scalar particles inside stars, followed by their emission, may constitute a powerful stellar cooling mechanism. If this process takes place at a high rate, it may alter 
star evolution, eventually leading to conflict with astrophysical observations [42]. This allows one to place strong constraints on the $\phi$ scalar couplings. The dominant cooling mechanisms are scalar bremsstrahlung in lepton-nucleus scattering, $\ell^{-}+N \rightarrow \ell^{-}+N+\phi$, and the Compton process $\gamma+\ell^{-} \rightarrow \ell^{-}+\phi$. Their relative importance depends on the density and temperature of the medium, and therefore on the astrophysical scenario. In particular, the Compton process dominates only at low densities and high temperatures, conditions that can be found in red giants. Limits can also be derived from the production of ultralight scalars in supernovae. The scalar $\phi$ can be efficiently produced and, since it will typically escape without interacting with the medium, a net transport of energy out of the supernova will take place. Such a loss of energy may dramatically affect other processes taking place in the supernova, such as neutrino production.

Plenty of works have recently studied the question of cooling by the emission of ultralight scalars in astrophysical scenarios [11, 39, 43-45]. However, to the best of our knowledge, all of them consider axions or ALPs. These are low-mass pseudoscalars and thus, their impact on stellar evolution can only be used to constrain pseudoscalar couplings. Even though we will not provide a detailed calculation to support this statement, we will argue that similar bounds can be set on the scalar couplings.

To make explicit the pure scalar and pseudoscalar interactions, we can use a redefinition of our Lagrangian in eq. (2.1) which, for the diagonal terms, can be written as

$$
\mathcal{L}_{\ell \ell \phi}^{\operatorname{diag}}=\phi \bar{\ell}_{\beta}\left(S^{\beta \beta} P_{L}+S^{\beta \beta *} P_{R}\right) \ell_{\beta}=\phi \bar{\ell}_{\beta}\left[\operatorname{Re} S^{\beta \beta}-i \operatorname{Im} S^{\beta \beta} \gamma_{5}\right] \ell_{\beta},
$$

with $S^{\beta \beta}=S_{L}^{\beta \beta}+S_{R}^{\beta \beta *}$. For a pure pseudoscalar, only $\operatorname{Im} S^{\beta \beta}$ is present.

The currently most stringent limit on the pseudoscalar coupling with electrons is obtained from white dwarfs. Specifically, the limit is obtained by considering the bremsstrahlung process, which can be very efficient in the dense core of a white dwarf. Using data from the Sloan Digital Sky Survey and the SuperCOSMOS Sky Survey, ref. [39] found (at 90\% C.L.)

$$
\operatorname{Im} S^{e e}<2.1 \times 10^{-13} .
$$

The coupling with muons has been recently studied in some works [39, 43, 44]. In this case the process ultimately used to set the contraint is neutrino production, clearly suppressed if energy is transported out of the supernova by scalars produced in $\mu+\gamma \rightarrow \mu+\phi$. Using the famous supernova SN1987A, ref. [44] has found

$$
\operatorname{Im} S^{\mu \mu}<2.1 \times 10^{-10} .
$$

Setting precise limits for the scalar parts of the couplings would imply the calculation of the cross sections and the energy-loss rates per unit mass, as required to perform a complete analysis. Instead, one can gauge the relevance of the bounds on the scalar couplings with the following arguments. First, we note that if the charged lepton mass is neglected, the scalar and pseudoscalar couplings contribute in exactly the same way to the relevant cross sections. This is, however, a bad approximation, due to the low energies involved in the astrophysical scenarios that set the limits. For this reason, one must keep the charged lepton 


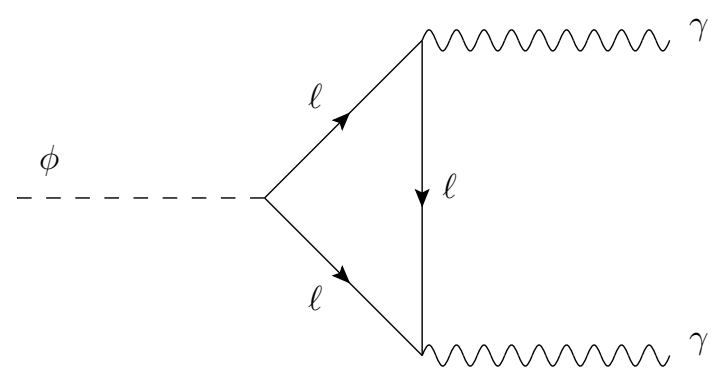

Figure 1. Loop induced coupling of $\phi$ to a pair of photons.

mass. We have numerically integrated the cross sections for a wide range of low energies and found that, for the same numerical value of $\operatorname{Re} S$ and $\operatorname{Im} S$, the scalar interaction always gives larger cross sections. Therefore, the constraints on the scalar couplings will be stronger and we can conclude that

$$
\operatorname{Re} S^{\beta \beta} \lesssim\left[\operatorname{Im} S^{\beta \beta}\right]_{\max }
$$

with $\beta=e, \mu$. Nevertheless, we point out that a detailed analysis of the cooling mechanism with pure scalars is required to fully determine the corresponding bounds.

Finally, one should note that these limits are based on the (reasonable) assumption that the scalar properties are not altered in the astrophysical medium. In particular, its mass and couplings are assumed to be the same as in vacuum. Some mechanisms have been recently proposed [46, 47] (see also previous work in [48]) that would make this assumption invalid. These works are mainly motivated by the recent XENON1T results, which include a $3.5 \sigma$ excess of low-energy electron recoil events [49]. An axion explaining this excess would violate the astrophysical constraints, since the required coupling to electrons would be larger than the limit in eq. (3.2), see for instance [45]. This motivates the consideration of mechanisms that alter the effective couplings to electrons or the axion mass in high density scenarios. If any of these mechanisms are at work, larger diagonal couplings would be allowed. However, we note that additional bounds, not derived from astrophysical observations, can be set on the diagonal couplings. This is precisely what we proceed to discuss.

\section{$3.2 \quad$ 1-loop coupling to photons}

The interaction of the scalar $\phi$ to a pair of photons is described by the effective Lagrangian

$$
\mathcal{L}_{\phi \gamma \gamma}=g_{S \gamma \gamma} \phi F_{\mu \nu} F^{\mu \nu}+g_{A \gamma \gamma} \phi F_{\mu \nu} \widetilde{F}^{\mu \nu}
$$

where $g_{S \gamma \gamma}$ and $g_{A \gamma \gamma}$ are the couplings for a pure scalar and a pure pseudoscalar, respectively, and $\widetilde{F}^{\mu \nu}$ is the dual electromagnetic tensor, defined as

$$
\widetilde{F}^{\mu \nu}=\frac{1}{2} \varepsilon^{\mu \nu \alpha \beta} F_{\alpha \beta} .
$$

The $g_{S \gamma \gamma}$ and $g_{A \gamma \gamma}$ couplings can be induced at the 1-loop level from diagrams involving charged leptons, as shown in figure 1. Since $g_{S \gamma \gamma}$ and $g_{A \gamma \gamma}$ are constrained by a variety 
of experimental sources, this can be used to set indirect constraints on the $\phi$ couplings to charged leptons introduced in eq. (2.1). In particular, we will take advantage of this relation to get additional limits on the lepton flavor conserving couplings of $\phi$.

The 1-loop analytical expression for $g_{S \gamma \gamma}$ and $g_{A \gamma \gamma}$ can be written as [50]

$$
\left|g_{I \gamma \gamma}\right|^{2}=\frac{\alpha^{2}}{64 \pi^{2}}\left|\sum_{\beta} \frac{g_{I \beta \beta}}{m_{\beta}} A_{1 / 2}^{I}\left(\tau_{\beta}\right)\right|^{2},
$$

where $I=S, A$ and we sum over $\beta=e, \mu, \tau$. Here $g_{I \beta \beta}$ denote the $\phi$ couplings to the charged leptons, and their relation to $S_{L}$ and $S_{R}$ is given below. $A_{1 / 2}^{S}$ and $A_{1 / 2}^{A}$ are 1-loop fermionic functions defined as

$$
A_{1 / 2}^{S}\left(\tau_{\beta}\right)=2\left[\tau_{\beta}+\left(\tau_{\beta}-1\right) f\left(\tau_{\beta}\right)\right] \tau_{\beta}^{-2}
$$

for the scalar coupling and

$$
A_{1 / 2}^{A}\left(\tau_{\beta}\right)=2 \tau_{\beta}^{-1} f\left(\tau_{\beta}\right)
$$

for the pseudoscalar case, with $\tau_{\beta}=m_{\phi}^{2} / 4 m_{\beta}^{2}$. The function $f(\tau)$ can be found for instance in [51]. It is given by

$$
f(\tau) \equiv\left\{\begin{array}{ll}
\arcsin ^{2} \sqrt{\tau} & \tau \leq 1 \\
-\frac{1}{4}\left[\log \frac{1+\sqrt{1-\tau^{-1}}}{1-\sqrt{1-\tau^{-1}}}-i \pi\right]^{2} & \tau>1
\end{array} .\right.
$$

In this work we consider the case of an ultralight scalar. In the massless limit, the loop functions reduce simply to $A_{1 / 2}^{S}(0)=\frac{4}{3}$ and $A_{1 / 2}^{A}(0)=2$, and then we can write

$$
\begin{aligned}
& \left|g_{S \gamma \gamma}\right|^{2}=\frac{\alpha^{2}}{36 \pi^{2}}\left|\sum_{\beta} \frac{g_{S \beta \beta}}{m_{\beta}}\right|^{2}, \\
& \left|g_{A \gamma \gamma}\right|^{2}=\frac{\alpha^{2}}{16 \pi^{2}}\left|\sum_{\beta} \frac{g_{A \beta \beta}}{m_{\beta}}\right|^{2},
\end{aligned}
$$

with the couplings to the charged leptons being given by

$$
\begin{aligned}
& g_{S \beta \beta}=\operatorname{Re} S^{\beta \beta}, \\
& g_{A \beta \beta}=\operatorname{Im} S^{\beta \beta} .
\end{aligned}
$$

We are now in position to compare to the current experimental limits on the coupling to photons. These are of two types. First, let us consider astrophysical limits. Magnetic fields around astrophysical sources of photons may transform these into scalars, an effect that can be used to set constraints on their coupling. Ref. [52] provides a comprehensive recollection of limits from astrophysical observations. Using results from [53], this reference finds that for scalar masses in the range $m_{\phi} \ll 1 \mathrm{peV}-1 \mathrm{neV}$, astrophysical constraints imply

$$
g_{I \gamma \gamma} \lesssim\left(10^{-12}-10^{-11}\right) \mathrm{GeV}^{-1}
$$


for both scalar and pseudoscalar couplings. Taking this into account, we can find the relations

$$
\begin{aligned}
& \left|\sum_{\beta} \frac{\operatorname{Re} S^{\beta \beta}}{m_{\beta}}\right|^{2}=\frac{36 \pi^{2}}{\alpha^{2}} g_{S \gamma \gamma}^{2}<6.7 \times 10^{-16} \mathrm{GeV}^{-2}, \\
& \left|\sum_{\beta} \frac{\operatorname{Im} S^{\beta \beta}}{m_{\beta}}\right|^{2}=\frac{16 \pi^{2}}{\alpha^{2}} g_{A \gamma \gamma}^{2}<3.0 \times 10^{-16} \mathrm{GeV}^{-2},
\end{aligned}
$$

which translate into very stringent bounds on the diagonal couplings to charged leptons, $S^{e e} \lesssim 10^{-11}$ and $S^{\mu \mu} \lesssim 10^{-9}$. The OSQAR experiment [54], a light-shining-through-a-wall experiment, has also derived limits for massless scalars. Again, these are valid for both scalar and pseudoscalar couplings,

$$
g_{I \gamma \gamma}<5.76 \times 10^{-8} \mathrm{GeV}^{-1},
$$

and therefore,

$$
\begin{aligned}
& \left|\sum_{\beta} \frac{\operatorname{Re} S^{\beta \beta}}{m_{\beta}}\right|^{2}=\frac{36 \pi^{2}}{\alpha^{2}} g_{S \gamma \gamma}^{2}<3.8 \times 10^{-8} \mathrm{GeV}^{-2}, \\
& \left|\sum_{\beta} \frac{\operatorname{Im} S^{\beta \beta}}{m_{\beta}}\right|^{2}=\frac{16 \pi^{2}}{\alpha^{2}} g_{A \gamma \gamma}^{2}<1.7 \times 10^{-8} \mathrm{GeV}^{-2} .
\end{aligned}
$$

These relations also imply strong contraints on the diagonal couplings to charged leptons, but milder than in the previous case, $S^{e e} \lesssim 10^{-7}$ and $S^{\mu \mu} \lesssim 10^{-5}$.

Finally, we point out that these indirect limits are strictly only valid if the diagrams in figure 1 are the only contribution to the $\phi$ coupling to photons. If more contributions exist, possible cancellations among them may reduce the total coupling so that the constraints are satisfied for larger couplings to charged leptons. We should also note that astrophysical constraints are subject to the same limitation discussed above. They rely on the assumption that the properties of $\phi$ in the astrophysical medium are the same as in vacuum.

\section{Leptonic observables}

\section{$4.1 \quad \ell_{\alpha} \rightarrow \ell_{\beta} \phi$}

The off-diagonal $S_{A}^{\beta \alpha}$ scalar couplings, with $A=L, R$, can be directly constrained by the LFV decays $\ell_{\alpha} \rightarrow \ell_{\beta} \phi$. Using the effective Lagrangian in eq. (2.1), it is straightforward to obtain

$$
\Gamma\left(\ell_{\alpha} \rightarrow \ell_{\beta} \phi\right)=\frac{m_{\alpha}}{32 \pi}\left(\left|S_{L}^{\beta \alpha}\right|^{2}+\left|S_{R}^{\beta \alpha}\right|^{2}\right),
$$

where terms proportional to the small ratio $m_{\beta} / m_{\alpha}$ have been neglected. ${ }^{1}$

\footnotetext{
${ }^{1}$ We must notice that this approximation is not equally good for all $\ell_{\alpha} \rightarrow \ell_{\beta} \phi$ cases. This is because the ratio $m_{\mu} / m_{\tau} \sim 0.1$ is not completely negligible. Therefore, while the approximation is very good for $\mu \rightarrow e \phi$ and $\tau \rightarrow e \phi$, it may lead to an error of the order of $20 \%$ in $\tau \rightarrow \mu \phi$. This deviation is acceptable, but can be accounted for by including additional terms proportional to $m_{\mu} / m_{\tau}$, hence leading to a much more complicated analytical expression. Completely analogous comments can be made for the rest of the observables discussed in this section.
} 


\section{$4.2 \quad \ell_{\alpha} \rightarrow \ell_{\beta} \gamma \phi$}

The decay width for the 3 -body LFV process $\ell_{\alpha} \rightarrow \ell_{\beta} \gamma \phi$ can be written as

$$
\Gamma\left(\ell_{\alpha} \rightarrow \ell_{\beta} \gamma \phi\right)=\frac{\alpha m_{\alpha}}{64 \pi^{2}}\left(\left|S_{L}^{\beta \alpha}\right|^{2}+\left|S_{R}^{\beta \alpha}\right|^{2}\right) \mathcal{I}\left(x_{\min }, y_{\min }\right),
$$

where terms proportional to $m_{\beta} / m_{\alpha}$ have been neglected. Here $\mathcal{I}\left(x_{\min }, y_{\min }\right)$ is a phase space integral given by

$$
\mathcal{I}\left(x_{\min }, y_{\min }\right)=\int \mathrm{d} x \mathrm{~d} y \frac{(x-1)(2-x y-y)}{y^{2}(1-x-y)},
$$

and we have introduced the usual dimensionless parameters $x$ and $y$, defined as

$$
x=\frac{2 E_{\beta}}{m_{\alpha}}, \quad y=\frac{2 E_{\gamma}}{m_{\alpha}},
$$

which, together with $z=2 E_{\phi} / m_{\alpha}$, must fulfill the kinematical condition $x+y+z=2$. We point out that our analytical results match those in [55], except for redefinitions in the couplings. ${ }^{2}$

The phase space integral in eq. (4.3) depends on $x_{\min }$ and $y_{\min }$, the minimal values that the $x$ and $y$ parameters may take. While one could naively think that these are just dictated by kinematics, they are actually determined by the minimal $\ell_{\beta}$ lepton and photon energies measured in a given experiment. This not only properly adapts the calculation of the phase space integral to the physical region explored in a real experiment, but also cures the kinematical divergences that would otherwise appear. In fact, we note that the integral in eq. (4.3) diverges when the photon energy vanishes $(y \rightarrow 0)$. This is the well-known infrared divergence that also appears, for instance, in the radiative SM decay $\mu \rightarrow e \nu \bar{\nu} \gamma$. Another divergence is encountered when the photon and the $\ell_{\beta}$ lepton in the final state are emitted in the same direction. The angle between their momenta is given by

$$
\cos \theta_{\beta \gamma}=1+\frac{2-2(x+y)}{x y} .
$$

Since we work in the limit $m_{\beta}=0$, one finds a colinear divergence in configurations in which the photon and the $\ell_{\beta}$ lepton have their momenta aligned $\left(\theta_{\beta \gamma} \rightarrow 0\right)$. However, any real experimental setup has a finite experimental resolution, which implies a non-zero minimum measurable $E_{\gamma}$ and a non-zero minimum $\theta_{\beta \gamma}$ angle. Therefore, by restricting the phase space integration to the kinematical region explored in a practical situation, all divergences disappear.

Direct comparison with eq. (4.1) allows one to establish the relation

$$
\Gamma\left(\ell_{\alpha} \rightarrow \ell_{\beta} \gamma \phi\right)=\frac{\alpha}{2 \pi} \mathcal{I}\left(x_{\min }, y_{\min }\right) \Gamma\left(\ell_{\alpha} \rightarrow \ell_{\beta} \phi\right),
$$

which tells us that $\ell_{\alpha} \rightarrow \ell_{\beta} \gamma \phi$ is suppressed with respect to $\ell_{\alpha} \rightarrow \ell_{\beta} \phi$ due to an additional $\alpha$ coupling and a phase space factor. In fact, the latter turns out to be the main source of suppression.

\footnotetext{
${ }^{2}$ In the model considered in [55], the right-handed coupling was suppressed and hence neglected.
} 


\section{$4.3 \quad \ell_{\alpha} \rightarrow \ell_{\beta} \gamma$}

The amplitude for the $\ell_{\alpha} \rightarrow \ell_{\beta} \gamma$ radiative decay only receives contributions from dipole operators and takes the general form

$$
\mathcal{M}_{\phi}=-e \bar{u}_{\beta}\left\{m_{\alpha} \sigma^{\mu \nu} q_{\nu}\left[\left(K_{2}^{L}\right)^{\beta \alpha} P_{L}+\left(K_{2}^{R}\right)^{\beta \alpha} P_{R}\right]\right\} u_{\alpha} \varepsilon_{\mu}^{*},
$$

where $u$ and $v$ are spinors and $q_{\mu}$ and $\varepsilon_{\mu}$ are the photon 4-momentum and polarization vector, respectively. The $K_{2}^{L}$ and $K_{2}^{R}$ coefficients are induced at the one-loop level, as shown in figure 2. We find the expressions

$$
\begin{aligned}
& \left(K_{2}^{L}\right)^{\beta \alpha}=\frac{C_{L}^{\gamma \alpha} C_{L}^{\beta \gamma} f_{1}\left(m_{\alpha}, m_{\beta}, m_{\gamma}\right)+C_{L}^{\gamma \alpha} C_{R}^{\beta \gamma} f_{2}\left(m_{\alpha}, m_{\beta}, m_{\gamma}\right)+C_{R}^{\gamma \alpha} C_{L}^{\beta \gamma} f_{2}\left(m_{\beta}, m_{\alpha}, m_{\gamma}\right)}{32 \pi^{2} m_{\alpha}^{5} m_{\beta}^{3}\left(m_{\alpha}+m_{\beta}\right)\left(m_{\alpha}-m_{\beta}\right)^{2}}, \\
& \left(K_{2}^{R}\right)^{\beta \alpha}=\frac{C_{R}^{\gamma \alpha} C_{R}^{\beta \gamma} f_{1}\left(m_{\beta}, m_{\alpha}, m_{\gamma}\right)+C_{L}^{\gamma \alpha} C_{R}^{\beta \gamma} f_{2}\left(m_{\beta}, m_{\alpha}, m_{\gamma}\right)+C_{R}^{\gamma \alpha} C_{L}^{\beta \gamma} f_{2}\left(m_{\alpha}, m_{\beta}, m_{\gamma}\right)}{32 \pi^{2} m_{\alpha}^{5} m_{\beta}^{3}\left(m_{\alpha}+m_{\beta}\right)\left(m_{\alpha}-m_{\beta}\right)^{2}},
\end{aligned}
$$

where a sum over $\gamma$ is implicit here and the $f_{i}$ loop functions are defined as

$$
\begin{aligned}
f_{1}\left(m_{\alpha}, m_{\beta}, m_{\gamma}\right)= & 2 m_{\alpha} m_{\beta} m_{\gamma}\left[m_{\beta}^{2}\left(m_{\alpha}^{2}-m_{\beta}^{2}\right)\left(m_{\alpha}^{2}-m_{\gamma}^{2}\right) \log \frac{m_{\gamma}^{2}}{m_{\gamma}^{2}-m_{\alpha}^{2}}\right. \\
& +m_{\alpha}^{2}\left(m_{\beta}^{2}-m_{\alpha}^{2}\right)\left(m_{\beta}^{2}-m_{\gamma}^{2}\right) \log \frac{m_{\gamma}^{2}}{m_{\gamma}^{2}-m_{\beta}^{2}} \\
& \left.+m_{\alpha}^{2} m_{\beta}^{2}\left(m_{\alpha}^{2}-m_{\beta}^{2}\right)^{2} C_{0}\left(0, m_{\alpha}^{2}, m_{\beta}^{2}, m_{\gamma}, m_{\gamma}, 0\right)\right] \\
f_{2}\left(m_{\alpha}, m_{\beta}, m_{\gamma}\right)= & -m_{\alpha}^{3} m_{\beta}^{2}\left(m_{\alpha}^{2}-m_{\beta}^{2}\right)\left(m_{\beta}^{2}+m_{\gamma}^{2}\right)-m_{\alpha} m_{\beta}^{4}\left(m_{\alpha}^{4}-m_{\gamma}^{4}\right) \log \frac{m_{\gamma}^{2}}{m_{\gamma}^{2}-m_{\alpha}^{2}} \\
& +m_{\alpha}^{3}\left(m_{\beta}^{2}-m_{\gamma}^{2}\right)\left[2 m_{\beta}^{2} m_{\gamma}^{2}+m_{\alpha}^{2}\left(m_{\beta}^{2}-m_{\gamma}^{2}\right)\right] \log \frac{m_{\gamma}^{2}}{m_{\gamma}^{2}-m_{\beta}^{2}} \\
& -2 m_{\alpha}^{3} m_{\beta}^{4} m_{\gamma}^{2}\left(m_{\alpha}^{2}-m_{\beta}^{2}\right) C_{0}\left(0, m_{\alpha}^{2}, m_{\beta}^{2}, m_{\gamma}, m_{\gamma}, 0\right),
\end{aligned}
$$

and we have introduced here the usual scalar Passarino-Veltman three-point function

$$
C_{0}\left(0, m_{\alpha}^{2}, m_{\beta}^{2}, m_{\gamma}, m_{\gamma}, 0\right)=\frac{1}{2\left(m_{\alpha}^{2}-m_{\beta}^{2}\right)}\left[\log ^{2}\left(-\frac{m_{\gamma}^{2}}{m_{\alpha}^{2}}\right)-\log ^{2}\left(-\frac{m_{\gamma}^{2}}{m_{\beta}^{2}}\right)+2 \operatorname{Li}_{2} \frac{m_{\gamma}^{2}}{m_{\alpha}^{2}}-2 \operatorname{Li}_{2} \frac{m_{\gamma}^{2}}{m_{\beta}^{2}}\right] .
$$

The $C_{L, R}$ couplings that appear in eqs. (4.8) and (4.9) are related to the $S_{L, R}$ couplings introduced in the effective Lagrangian in eq. (2.1). The relation depends on the particular diagram under consideration:

$$
C_{L}^{\eta \rho}=\left\{\begin{array}{l}
S_{L}^{\eta \rho} m_{\eta}<m_{\rho} \\
S_{R}^{\rho \eta *} m_{\eta}>m_{\rho} \\
S^{\eta \eta} \quad \eta=\rho
\end{array}\right.
$$




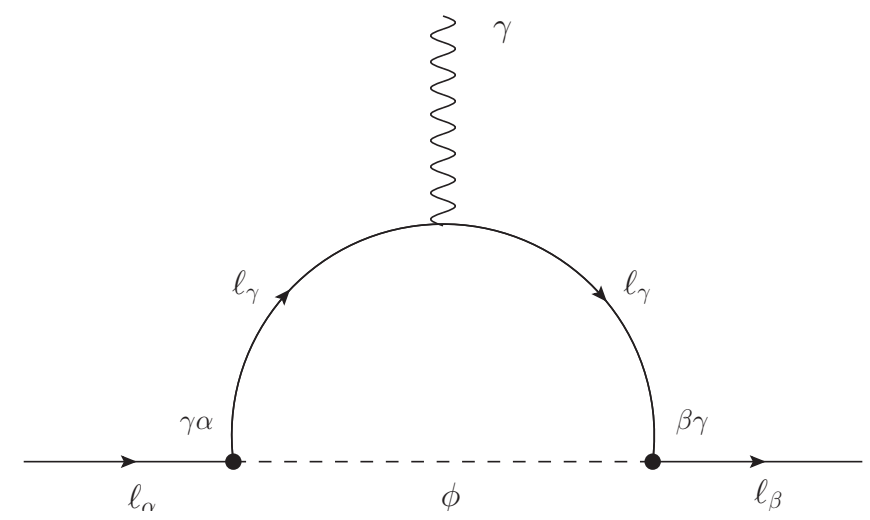

Figure 2. One-loop Feynman diagram contributing to the process $\ell_{\alpha} \rightarrow \ell_{\beta} \gamma$ described by the effective Lagrangian in eq. (2.1). The flavor indices of the couplings contributing to the diagram have been specified in the vertices.

and

$$
C_{R}^{\eta \rho}=\left\{\begin{array}{cc}
S_{R}^{\eta \rho} & m_{\eta}<m_{\rho} \\
S_{L}^{\rho \eta *} & m_{\eta}>m_{\rho} \\
S^{\eta \eta *} & \eta=\rho
\end{array} .\right.
$$

It proves convenient to find approximate expressions for the $K_{2}^{L, R}$ coefficients, obtained at leading order in $m_{\beta}$. We find

$$
\left(K_{2}^{L}\right)^{\beta \alpha}=\frac{S^{\beta \beta} S_{R}^{\beta \alpha}}{32 \pi^{2} m_{\alpha}^{2}}-\frac{S_{L}^{\beta \alpha}\left[S^{\alpha \alpha}\left(\pi^{2}-6\right)+S^{\alpha \alpha *}\left(\pi^{2}-9\right)\right]}{96 \pi^{2} m_{\alpha}^{2}}+\frac{1}{32 \pi^{2} m_{\alpha}}\left\{\begin{array}{cc}
\frac{S_{L}^{\beta \gamma} S_{R}^{\gamma \alpha}}{m_{\alpha}} & m_{\alpha} \gg m_{\gamma} \gg m_{\beta} \\
\frac{S_{R}^{\gamma *} S_{R}^{\gamma \alpha}}{m_{\alpha}} & m_{\alpha} \gg m_{\beta} \gg m_{\gamma} \\
-\frac{S_{L}^{\beta \gamma} S_{R}^{\alpha \gamma *}}{m_{\gamma}} & m_{\gamma} \gg m_{\alpha}
\end{array}\right.
$$

and

$$
\left(K_{2}^{R}\right)^{\beta \alpha}=\frac{S^{\beta \beta *} S_{L}^{\beta \alpha}}{32 \pi^{2} m_{\alpha}^{2}}-\frac{S_{R}^{\beta \alpha}\left[S^{\alpha \alpha}\left(\pi^{2}-9\right)+S^{\alpha \alpha *}\left(\pi^{2}-6\right)\right]}{96 \pi^{2} m_{\alpha}^{2}}+\frac{1}{32 \pi^{2} m_{\alpha}}\left\{\begin{array}{cc}
\frac{S_{R}^{\beta \gamma} S_{L}^{\gamma \alpha}}{m_{\alpha}} & m_{\alpha} \gg m_{\gamma} \gg m_{\beta} \\
\frac{S_{L}^{\gamma \beta *} S_{L}^{\gamma \alpha}}{m_{\alpha}^{\alpha}} & m_{\alpha} \gg m_{\beta} \gg m_{\gamma}, \\
-\frac{S_{R}^{\beta \gamma} S_{L}^{\alpha \gamma *}}{m_{\gamma}} & m_{\gamma} \gg m_{\alpha}
\end{array}\right.
$$

We note, however, that these approximate expressions may only serve as an estimate for the order of magnitude of the $K_{2}^{L, R}$ coefficients, since large errors $(\sim 50 \%)$ are obtained in some cases due to the appearance of large logs. Finally, upon substitution in eq. (2.5), one obtains the total decay width of the process. ${ }^{3}$ Then, we can compare our analytical results with those found in [32]. Assuming that the only non-vanishing couplings are the ones involving the $\mu \mu$ and $e \mu$ flavor combinations, and making the replacements

$$
S^{\mu \mu}=i \frac{m_{\mu} c_{\mu \mu}}{f}, \quad S_{L}^{e \mu}=i \frac{m_{\mu}\left(k_{e}\right)_{e \mu}}{f}, \quad S_{R}^{e \mu}=i \frac{m_{\mu}\left(k_{E}\right)_{e \mu}}{f},
$$

full agreement is recovered.

\footnotetext{
${ }^{3}$ For completeness, we note that the expression for the $\ell_{\alpha} \rightarrow \ell_{\beta} \gamma$ decay width without neglecting $m_{\beta}^{2}$ is $\Gamma\left(\ell_{\alpha} \rightarrow \ell_{\beta} \gamma\right)=\frac{e^{2}\left(m_{\alpha}^{2}-m_{\beta}^{2}\right)^{3}}{16 \pi m_{\alpha}}\left[\left|\left(K_{2}^{L}\right)^{\beta \alpha}\right|^{2}+\left|\left(K_{2}^{R}\right)^{\beta \alpha}\right|^{2}\right]$. 

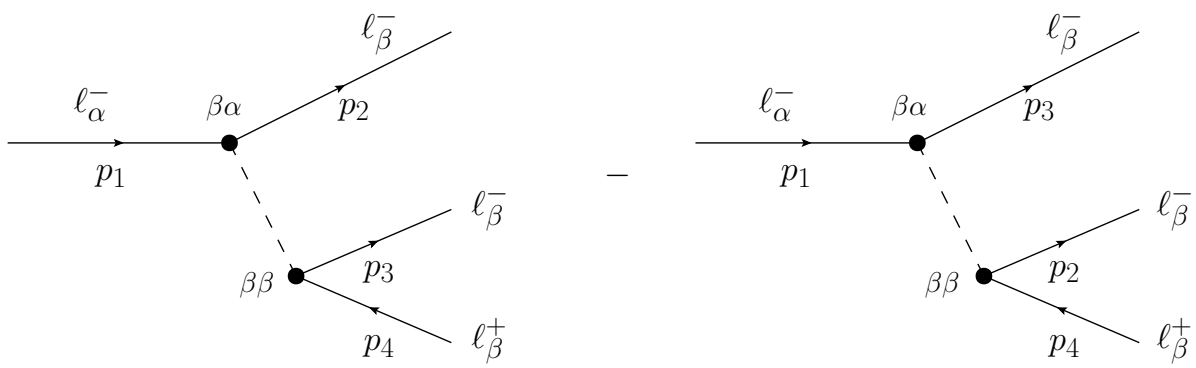

Figure 3. Tree-level Feynman diagrams contributing to the process $\ell_{\alpha}^{-} \rightarrow \ell_{\beta}^{-} \ell_{\beta}^{-} \ell_{\beta}^{+}$described by the effective Lagrangian in eq. (2.1).

\section{$4.4 \quad \ell_{\alpha}^{-} \rightarrow \ell_{\beta}^{-} \ell_{\beta}^{-} \ell_{\beta}^{+}$}

Complete expressions for the $\ell_{\alpha}^{-} \rightarrow \ell_{\beta}^{-} \ell_{\beta}^{-} \ell_{\beta}^{+}$decay width in the absence of $\phi$ can be found in [56]. Here we are interested in the new contributions mediated by the scalar $\phi$, which are given by the Feynman diagrams shown in figure 3. It is straightforward to derive the associated amplitude, given by

$$
\begin{aligned}
\mathcal{M}_{\phi}= & \bar{u}\left(p_{3}\right) i\left(S^{\beta \beta} P_{L}+S^{\beta \beta *} P_{R}\right) v\left(p_{4}\right) \frac{i}{q^{2}+i \varepsilon} \bar{u}\left(p_{2}\right) i\left(S_{L}^{\beta \alpha} P_{L}+S_{R}^{\beta \alpha} P_{R}\right) u\left(p_{1}\right) \\
& -\bar{u}\left(p_{2}\right) i\left(S^{\beta \beta} P_{L}+S^{\beta \beta *} P_{R}\right) v\left(p_{4}\right) \frac{i}{k^{2}+i \varepsilon} \bar{u}\left(p_{3}\right) i\left(S_{L}^{\beta \alpha} P_{L}+S_{R}^{\beta \alpha} P_{R}\right) u\left(p_{1}\right) .
\end{aligned}
$$

Here $q=p_{1}-p_{2}$ and $k=p_{1}-p_{3}$ are the $\phi$ virtual momenta and we have explicitly indicated the flavor indices of the $S_{L, R}$ coefficients. The total decay width can then be written as

$$
\Gamma\left(\ell_{\alpha}^{-} \rightarrow \ell_{\beta}^{-} \ell_{\beta}^{-} \ell_{\beta}^{+}\right)=\Gamma_{\bar{\phi}}\left(\ell_{\alpha}^{-} \rightarrow \ell_{\beta}^{-} \ell_{\beta}^{-} \ell_{\beta}^{+}\right)+\Gamma_{\phi}\left(\ell_{\alpha}^{-} \rightarrow \ell_{\beta}^{-} \ell_{\beta}^{-} \ell_{\beta}^{+}\right),
$$

where $\Gamma_{\bar{\phi}}$ is the decay width in the absence of $\phi$, given in [56], and

$$
\begin{aligned}
& \Gamma_{\phi}\left(\ell_{\alpha}^{-} \rightarrow \ell_{\beta}^{-} \ell_{\beta}^{-} \ell_{\beta}^{+}\right)= \\
& \quad \frac{m_{\alpha}}{512 \pi^{3}}\left\{\left(\left|S_{L}^{\beta \alpha}\right|^{2}+\left|S_{R}^{\beta \alpha}\right|^{2}\right)\left\{\left|S^{\beta \beta}\right|^{2}\left(4 \log \frac{m_{\alpha}}{m_{\beta}}-\frac{49}{6}\right)-\frac{2}{6}\left[\left(S^{\beta \beta *}\right)^{2}+\left(S^{\beta \beta}\right)^{2}\right]\right\}\right. \\
& \quad-\frac{m_{\alpha}^{2}}{6}\left\{S_{L}^{\beta \alpha} S^{\beta \beta} A_{L L}^{S *}+2 S_{L}^{\beta \alpha} S^{\beta \beta *} A_{L R}^{S *}+2 S_{R}^{\beta \alpha} S^{\beta \beta} A_{R L}^{S *}+S_{R}^{\beta \alpha} S^{\beta \beta *} A_{R R}^{S *}\right. \\
& \quad-12\left(S_{L}^{\beta \alpha} S^{\beta \beta} A_{L L}^{T *}+S_{R}^{\beta \alpha} S^{\beta \beta *} A_{R R}^{T *}\right)-4\left(S_{R}^{\beta \alpha} S^{\beta \beta} A_{R L}^{V *}+S_{L}^{\beta \alpha} S^{\beta \beta *} A_{L R}^{V *}\right) \\
& \left.\left.+6 e^{2}\left[S_{R}^{\beta \alpha} S^{\beta \beta}\left(K_{2}^{L}\right)^{\beta \alpha *}+S_{L}^{\beta \alpha} S^{\beta \beta *}\left(K_{2}^{R}\right)^{\beta \alpha *}\right]+\text { c.c. }\right\}\right\},
\end{aligned}
$$

where in this expression $A_{X Y}^{I}=\left(A_{X Y}^{I}\right)^{\beta \beta \beta \alpha}$. In writing eq. (4.20) we have only kept the lowest order terms in powers of $m_{\beta}$ for each possible combination of couplings. This is equivalent to 0th order for all terms, with the exception of the ones in the first line, where the factor $\log \frac{m_{\alpha}}{m_{\beta}}$ avoids the appearance of an infrared divergence. An expression including terms up to first order in $m_{\beta}$ is given in appendix A. 

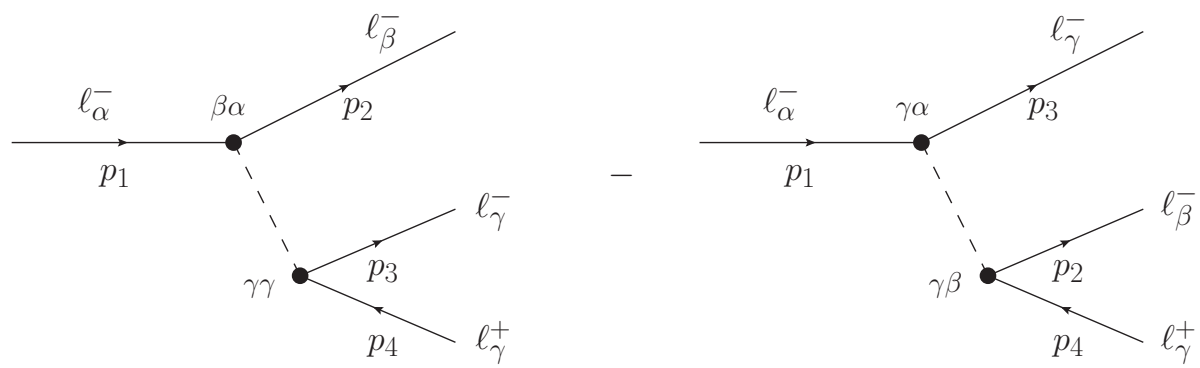

Figure 4. Tree-level Feynman diagrams contributing to the process $\ell_{\alpha}^{-} \rightarrow \ell_{\beta}^{-} \ell_{\gamma}^{-} \ell_{\gamma}^{+}$described by the effective Lagrangian in eq. (2.1).

\section{$4.5 \quad \ell_{\alpha}^{-} \rightarrow \ell_{\beta}^{-} \ell_{\gamma}^{-} \ell_{\gamma}^{+}$}

Again, complete expressions for the $\ell_{\alpha}^{-} \rightarrow \ell_{\beta}^{-} \ell_{\gamma}^{-} \ell_{\gamma}^{+}$decay width in the absence of $\phi$ can be found in [56]. The new contributions mediated by the scalar $\phi$ are obtained from the Feynman diagrams shown in figure 4 . While the diagram on the left involves a flavor conserving $(\gamma \gamma)$ and a flavor violating $(\beta \alpha)$ vertex, both vertices in the diagram on the right violate flavor $(\gamma \alpha$ and $\gamma \beta)$. The associated amplitude is slightly different from that of the previous process and is given by

$$
\begin{aligned}
\mathcal{M}_{\phi}= & \bar{u}\left(p_{3}\right) i\left(S^{\gamma \gamma} P_{L}+S^{\gamma \gamma *} P_{R}\right) v\left(p_{4}\right) \frac{i}{q^{2}+i \varepsilon} \bar{u}\left(p_{2}\right) i\left(S_{L}^{\beta \alpha} P_{L}+S_{R}^{\beta \alpha} P_{R}\right) u\left(p_{1}\right) \\
& -\bar{u}\left(p_{2}\right) i\left(S_{L}^{\gamma \beta} P_{L}+S_{R}^{\gamma \beta} P_{R}\right) v\left(p_{4}\right) \frac{i}{k^{2}+i \varepsilon} \bar{u}\left(p_{3}\right) i\left(S_{L}^{\gamma \alpha} P_{L}+S_{R}^{\gamma \alpha} P_{R}\right) u\left(p_{1}\right) .
\end{aligned}
$$

Finally, the total decay width can be written as

$$
\Gamma\left(\ell_{\alpha}^{-} \rightarrow \ell_{\beta}^{-} \ell_{\gamma}^{-} \ell_{\gamma}^{+}\right)=\Gamma_{\bar{\phi}}\left(\ell_{\alpha}^{-} \rightarrow \ell_{\beta}^{-} \ell_{\gamma}^{-} \ell_{\gamma}^{+}\right)+\Gamma_{\phi}\left(\ell_{\alpha}^{-} \rightarrow \ell_{\beta}^{-} \ell_{\gamma}^{-} \ell_{\gamma}^{+}\right)
$$

where $\Gamma_{\bar{\phi}}$ is the decay width in the absence of $\phi$, given in [56], and

$$
\begin{aligned}
& \Gamma_{\phi}\left(\ell_{\alpha}^{-} \rightarrow \ell_{\beta}^{-} \ell_{\gamma}^{-} \ell_{\gamma}^{+}\right)= \\
& \quad \frac{m_{\alpha}}{512 \pi^{3}}\left\{\left(\left|S_{L}^{\beta \alpha}\right|^{2}+\left|S_{R}^{\beta \alpha}\right|^{2}\right)\left\{\left|S^{\gamma \gamma}\right|^{2}\left(4 \log \frac{m_{\alpha}}{m_{\gamma}}-\frac{23}{3}\right)-\frac{1}{3}\left[\left(S^{\gamma \gamma *}\right)^{2}+\left(S^{\gamma \gamma}\right)^{2}\right]\right\}\right. \\
& \quad+\left(\left|S_{L}^{\gamma \alpha}\right|^{2}+\left|S_{R}^{\gamma \alpha}\right|^{2}\right)\left(\left|S_{L}^{\gamma \beta}\right|^{2}+\left|S_{R}^{\gamma \beta}\right|^{2}\right)\left(2 \log \frac{m_{\alpha}}{m_{f}^{\max }}-3\right) \\
& \quad-\frac{1}{2}\left[S^{\gamma \gamma}\left(S_{L}^{\beta \alpha} S_{L}^{\gamma \alpha *} S_{L}^{\gamma \beta}+S_{R}^{\beta \alpha *} S_{R}^{\gamma \alpha} S_{R}^{\gamma \beta}\right)+\text { c.c. }\right] \\
& \quad+\frac{m_{\alpha}^{2}}{6}\left\{S_{L}^{\gamma \alpha} S_{L}^{\gamma \beta} A_{L L}^{S *}+S_{R}^{\gamma \alpha} S_{R}^{\gamma \beta} A_{R R}^{S *}-2 S^{\gamma \gamma}\left(S_{L}^{\beta \alpha} A_{L L}^{S *}+S_{L}^{\beta \alpha *} A_{L R}^{S}+S_{R}^{\beta \alpha} A_{R L}^{S *}+S_{R}^{\beta \alpha *} A_{R R}^{S}\right)\right. \\
& \quad+4\left(S_{L}^{\gamma \alpha} S_{R}^{\gamma \beta} A_{L R}^{V *}+S_{R}^{\gamma \alpha} S_{L}^{\gamma \beta} A_{R L}^{V *}\right)+12\left(S_{L}^{\gamma \alpha} S_{L}^{\gamma \beta} A_{L L}^{T *}+S_{R}^{\gamma \alpha} S_{R}^{\gamma \beta} A_{R R}^{T *}\right) \\
& \left.\left.\quad-6 e^{2}\left[S_{L}^{\gamma \alpha} S_{R}^{\gamma \beta}\left(K_{2}^{R}\right){ }^{\beta \alpha *}+S_{R}^{\gamma \alpha} S_{L}^{\gamma \beta}\left(K_{2}^{L}\right)^{\beta \alpha *}\right]+\text { c.c. }\right\}\right\},
\end{aligned}
$$



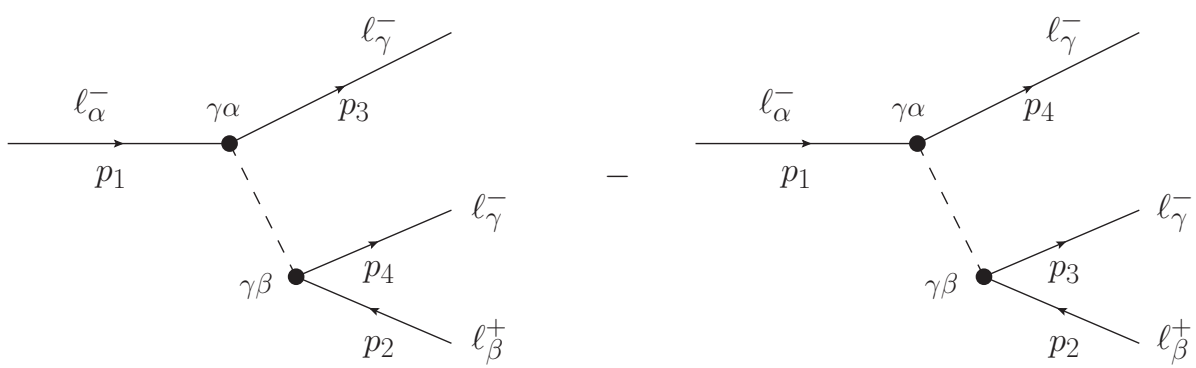

Figure 5. Tree-level Feynman diagrams contributing to the process $\ell_{\alpha}^{-} \rightarrow \ell_{\beta}^{+} \ell_{\gamma}^{-} \ell_{\gamma}^{-}$described by the effective Lagrangian in eq. (2.1).

where in this expression $A_{X Y}^{I}=\left(A_{X Y}^{I}\right)^{\gamma \gamma \beta \alpha}$. Also here $m_{f}^{\max }=\max \left(m_{\beta}, m_{\gamma}\right)$ and then the expression depends on the process in question. Once again, we have only kept the lowest order terms in powers of $m_{\beta}$ and $m_{\gamma}$ for each possible combination of couplings.

\section{6 $\quad \ell_{\alpha}^{-} \rightarrow \ell_{\beta}^{+} \ell_{\gamma}^{-} \ell_{\gamma}^{-}$}

Also for this process, complete expressions for the $\ell_{\alpha}^{-} \rightarrow \ell_{\beta}^{+} \ell_{\gamma}^{-} \ell_{\gamma}^{-}$decay width in the absence of $\phi$ can be found in [56]. The new contributions mediated by the scalar $\phi$ are given by the Feynman diagrams shown in figure 5 . We note that both vertices are necessarily flavor violating. The associated amplitude is given in this case by

$$
\begin{aligned}
\mathcal{M}_{\phi}= & \bar{u}\left(p_{4}\right) i\left(S_{L}^{\gamma \beta} P_{L}+S_{R}^{\gamma \beta} P_{R}\right) v\left(p_{2}\right) \frac{i}{q^{2}+i \varepsilon} \bar{u}\left(p_{3}\right) i\left(S_{L}^{\gamma \alpha} P_{L}+S_{R}^{\gamma \alpha} P_{R}\right) u\left(p_{1}\right) \\
& -\bar{u}\left(p_{3}\right) i\left(S_{L}^{\gamma \beta} P_{L}+S_{R}^{\gamma \beta} P_{R}\right) v\left(p_{2}\right) \frac{i}{k^{2}+i \varepsilon} \bar{u}\left(p_{4}\right) i\left(S_{L}^{\gamma \alpha} P_{L}+S_{R}^{\gamma \alpha} P_{R}\right) u\left(p_{1}\right) .
\end{aligned}
$$

Here $q=p_{1}-p_{3}$ and $k=p_{1}-p_{4}$ are different from their definitions in the processes above. Writing one more time the decay width as the sum of two contributions,

$$
\Gamma\left(\ell_{\alpha}^{-} \rightarrow \ell_{\beta}^{+} \ell_{\gamma}^{-} \ell_{\gamma}^{-}\right)=\Gamma_{\bar{\phi}}\left(\ell_{\alpha}^{-} \rightarrow \ell_{\beta}^{+} \ell_{\gamma}^{-} \ell_{\gamma}^{-}\right)+\Gamma_{\phi}\left(\ell_{\alpha}^{-} \rightarrow \ell_{\beta}^{+} \ell_{\gamma}^{-} \ell_{\gamma}^{-}\right),
$$

where $\Gamma_{\bar{\phi}}$ is the decay width in the absence of $\phi$, given in [56], we find that

$$
\begin{aligned}
& \Gamma_{\phi}\left(\ell_{\alpha}^{-} \rightarrow \ell_{\beta}^{+} \ell_{\gamma}^{-} \ell_{\gamma}^{-}\right)= \\
& \quad \frac{m_{\alpha}}{512 \pi^{3}}\left\{\left(\left|S_{L}^{\gamma \alpha}\right|^{2}+\left|S_{R}^{\gamma \alpha}\right|^{2}\right)\left(\left|S_{L}^{\gamma \beta}\right|^{2}+\left|S_{R}^{\gamma \beta}\right|^{2}\right)\left(2 \log \frac{m_{\alpha}}{m_{f}^{\max }}-3\right)\right. \\
& \quad-\frac{1}{2}\left(\left|S_{L}^{\gamma \alpha}\right|^{2}\left|S_{L}^{\gamma \beta}\right|^{2}+\left|S_{R}^{\gamma \alpha}\right|^{2}\left|S_{R}^{\gamma \beta}\right|^{2}\right) \\
& \quad+\frac{m_{\alpha}^{2}}{6}\left[-S_{L}^{\gamma \alpha} S_{L}^{\gamma \beta} A_{L L}^{S *}-S_{R}^{\gamma \alpha} S_{R}^{\gamma \beta} A_{R R}^{S *}-2\left(S_{L}^{\gamma \alpha} S_{R}^{\gamma \beta} A_{R L}^{S *}+S_{R}^{\gamma \alpha} S_{L}^{\gamma \beta} A_{L R}^{S *}\right)\right. \\
& \left.\left.\quad+4\left(S_{L}^{\gamma \alpha} S_{R}^{\gamma \beta} A_{R L}^{V *}+S_{R}^{\gamma \alpha} S_{L}^{\gamma \beta} A_{L R}^{V *}\right)+12\left(S_{L}^{\gamma \alpha} S_{L}^{\gamma \beta} A_{L L}^{T *}+S_{R}^{\gamma \alpha} S_{R}^{\gamma \beta} A_{R R}^{T *}\right)+\text { c.c. }\right]\right\},
\end{aligned}
$$

where in this expression $A_{X Y}^{I}=\left(A_{X Y}^{I}\right)^{\gamma \beta \gamma \alpha}$ and $m_{f}^{\max }=\max \left(m_{\beta}, m_{\gamma}\right)$. 


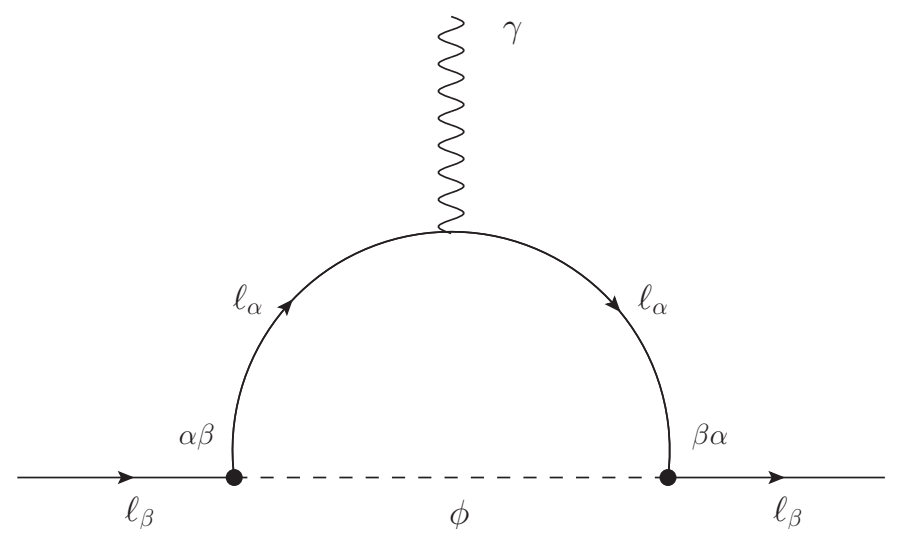

Figure 6. Feynman diagram for the one-loop contribution to the anomalous magnetic moment of charged leptons given by the interaction in eq. (2.1). The flavor indices of the couplings contributing to the diagram have been specified in the vertices. The relation between these couplings, which we generically denote as $C_{L, R}$ and $\widetilde{C}_{L, R}$, and the $S_{L, R}$ couplings in the effective Lagrangian of eq. (2.1) depends on the flavor states involved in the diagram. See text for a detailed explanation.

\subsection{Lepton magnetic and electric dipole moments}

We finally consider the magnetic and electric dipole moments of the charged leptons. These can be described by the effective Lagrangians

$$
\begin{aligned}
\mathcal{L}_{\mathrm{AMM}} & =\frac{e}{2 m_{\alpha}} a_{\alpha} \bar{\ell}_{\alpha} \sigma^{\mu \nu} F_{\mu \nu} \ell_{\alpha}, \\
\mathcal{L}_{\mathrm{EDM}} & =-\frac{i}{2} d_{\alpha} \bar{\ell}_{\alpha} \sigma^{\mu \nu} F_{\mu \nu} \gamma_{5} \ell_{\alpha} .
\end{aligned}
$$

The charged lepton dipole moments receive contributions mediated by the scalar $\phi$, as shown in figure $6 .{ }^{4}$ In the following we denote the chiral couplings in the $\bar{\ell}_{\alpha}-\ell_{\beta}-\phi$ vertex as $C_{L}^{\alpha \beta}$ and $C_{R}^{\alpha \beta}$, whereas the chiral couplings in the $\bar{\ell}_{\beta}-\ell_{\alpha}-\phi$ vertex are denoted as $\widetilde{C}_{L}^{\beta \alpha}$ and $\widetilde{C}_{R}^{\beta \alpha}$. The $C_{L, R}$ and $\widetilde{C}_{L, R}$ couplings are obviously related to the $S_{L, R}$ couplings in the effective Lagrangian of eq. (2.1), but this relation depends on the flavor states involved in the diagram, as discussed below. The amplitude associated to the diagram in figure 6 can be written as

$$
\begin{aligned}
i \mathcal{M}= & \int \frac{\mathrm{d}^{4} q}{(2 \pi)^{4}} \bar{u}_{\ell}\left(p^{\prime}, m_{\alpha}\right)\left[i\left(C_{L}^{\alpha \beta} P_{L}+C_{R}^{\alpha \beta} P_{R}\right)\right] \frac{i\left(\not p^{\prime}+\not p+m_{\beta}\right)}{\left(p^{\prime}+q\right)^{2}-m_{\beta}^{2}}\left(-i e \gamma^{\mu}\right) \frac{i\left(\not p+\not q+m_{\beta}\right)}{(p+q)^{2}-m_{\beta}^{2}} \\
& {\left[i\left(\widetilde{C}_{L}^{\beta \alpha} P_{L}+\widetilde{C}_{R}^{\beta \alpha} P_{R}\right)\right] \frac{i}{q^{2}} u_{\ell}\left(p, m_{\alpha}\right) \varepsilon_{\mu}^{*}(k), }
\end{aligned}
$$

where $m_{\alpha}$ and $m_{\beta}$ are the masses of the external and internal leptons, respectively, and we sum over the index $\beta$. One must now compare to the equivalent amplitude obtained

\footnotetext{
${ }^{4}$ Two-loop Barr-Zee contributions [57] to the charged leptons AMMs and EDMs can also be considered. However, while these might be relevant in some cases, we will assume that the $S_{L, R}$ couplings can at most have mild hierarchies among different flavors, hence making them numerically irrelevant with respect to the one-loop contributions considered here.
} 
with the effective Lagrangians in eqs. (4.27) and (4.28). After some algebra, one finds that the scalar $\phi$ induces the contributions to the anomalous magnetic moments (AMMs) and electric dipole moments (EDMs) of the charged leptons

$$
\begin{aligned}
\Delta a_{\alpha}= & \frac{1}{32 \pi^{2} m_{\alpha}^{4}}\left\{2 m_{\alpha} m_{\beta}\left[m_{\alpha}^{2}+\left(m_{\alpha}^{2}-m_{\beta}^{2}\right) \log \frac{m_{\beta}^{2}}{\left|m_{\beta}^{2}-m_{\alpha}^{2}\right|}\right]\left(C_{L}^{\alpha \beta} \widetilde{C}_{L}^{\beta \alpha}+C_{R}^{\alpha \beta} \widetilde{C}_{R}^{\beta \alpha}\right)\right. \\
& \left.-\left[m_{\alpha}^{2}\left(m_{\alpha}^{2}-2 m_{\beta}^{2}\right)-2 m_{\beta}^{2}\left(m_{\alpha}^{2}-m_{\beta}^{2}\right) \log \frac{m_{\beta}^{2}}{\left|m_{\beta}^{2}-m_{\alpha}^{2}\right|}\right]\left(C_{L}^{\alpha \beta} \widetilde{C}_{R}^{\beta \alpha}+C_{R}^{\alpha \beta} \widetilde{C}_{L}^{\beta \alpha}\right)\right\},
\end{aligned}
$$

and

$$
d_{\alpha}=\frac{i e m_{\beta}}{32 \pi^{2} m_{\alpha}^{4}}\left(C_{L}^{\alpha \beta} \widetilde{C}_{L}^{\beta \alpha}-C_{R}^{\alpha \beta} \widetilde{C}_{R}^{\beta \alpha}\right)\left[m_{\alpha}^{2}+\left(m_{\alpha}^{2}-m_{\beta}^{2}\right) \log \frac{m_{\beta}^{2}}{\left|m_{\beta}^{2}-m_{\alpha}^{2}\right|}\right] .
$$

These analytical results have been checked with the help of Package-X [58]. We note again that a sum over the index $\beta$ is performed in eqs. (4.30) and (4.31). Therefore, they include both flavor diagonal as well as flavor off-diagonal contributions to the dipole moments. We now consider these contributions separately and study their behavior in specific limits:

1. Flavor off-diagonal contribution with $m_{\beta} \ll m_{\alpha}$.

In this case the $C_{L, R}$ and $\widetilde{C}_{L, R}$ couplings are related to the $S_{L, R}$ couplings in eq. (2.1) as $\widetilde{C}_{L, R}^{\beta \alpha}=S_{L, R}^{\beta \alpha}$ and $C_{L, R}^{\alpha \beta}=S_{R, L}^{\beta \alpha *}$ and the expressions simplify to

$$
\begin{aligned}
\Delta a_{\alpha}= & \frac{1}{32 \pi^{2} m_{\alpha}}\left[-m_{\alpha}\left(\left|S_{L}^{\beta \alpha}\right|^{2}+\left|S_{R}^{\beta \alpha}\right|^{2}\right)\right. \\
& \left.+4 m_{\beta} \operatorname{Re}\left(S_{R}^{\beta \alpha} S_{L}^{\beta \alpha *}\right)\left(1+\log \frac{m_{\beta}^{2}}{m_{\alpha}^{2}}\right)\right]+\mathcal{O}\left(m_{\beta}^{2}\right)
\end{aligned}
$$

and

$$
d_{\alpha}=\frac{e m_{\beta}}{16 \pi^{2} m_{\alpha}^{2}} \operatorname{Im}\left(S_{R}^{\beta \alpha} S_{L}^{\beta \alpha *}\right)\left(1+\log \frac{m_{\beta}^{2}}{m_{\alpha}^{2}}\right)+\mathcal{O}\left(m_{\beta}^{3}\right)
$$

2. Flavor off-diagonal contribution with $m_{\beta} \gg m_{\alpha}$.

In this case the generic $C_{L, R}$ and $\widetilde{C}_{L, R}$ couplings are related to the $S_{L, R}$ couplings as $\widetilde{C}_{L, R}^{\beta \alpha}=S_{R, L}^{\alpha \beta *}$ and $C_{L, R}^{\alpha \beta}=S_{L, R}^{\alpha \beta}$, giving us

$$
\Delta a_{\alpha}=\frac{m_{\alpha}}{16 \pi^{2} m_{\beta}}\left[\operatorname{Re}\left(S_{R}^{\alpha \beta} S_{L}^{\alpha \beta *}\right)+\frac{m_{\alpha}}{6 m_{\beta}}\left(\left|S_{L}^{\beta \alpha}\right|^{2}+\left|S_{R}^{\beta \alpha}\right|^{2}\right)\right]+\mathcal{O}\left(m_{\alpha}^{3}\right),
$$

and

$$
d_{\alpha}=\frac{e}{32 \pi^{2} m_{\beta}} \operatorname{Im}\left(S_{R}^{\alpha \beta} S_{L}^{\alpha \beta *}\right)+\mathcal{O}\left(m_{\alpha}^{2}\right)
$$


3. Flavor diagonal contribution, i.e. $m_{\beta}=m_{\alpha}$.

Finally, in this case we have $C_{L}^{\alpha \alpha}=\widetilde{C}_{L}^{\alpha \alpha}=S^{\alpha \alpha}$ and $C_{R}^{\alpha \alpha}=\widetilde{C}_{R}^{\alpha \alpha}=S^{\alpha \alpha *}$, and we find the simple expression

$$
\Delta a_{\alpha}=\frac{1}{16 \pi^{2}}\left[3\left(\operatorname{Re} S^{\alpha \alpha}\right)^{2}-\left(\operatorname{Im} S^{\alpha \alpha}\right)^{2}\right]
$$

for the AMM of the charged lepton $\ell_{\alpha}$, which agrees with previous results in the literature. In particular, it matches exactly the expression given in [59] in the limit of a massless scalar, with the equivalence

$$
-\frac{m_{\alpha}}{v} a_{\alpha}^{S}=\frac{1}{2}\left(S^{\alpha \alpha}+S^{\alpha \alpha *}\right), \quad-i \frac{m_{\alpha}}{v} b_{\alpha}^{S}=-\frac{1}{2}\left(S^{\alpha \alpha}-S^{\alpha \alpha *}\right) .
$$

Regarding the expression for the EDM, it also acquires a very simple form in this case,

$$
d_{\alpha}=-\frac{e}{8 \pi^{2} m_{\alpha}}\left(\operatorname{Re} S^{\alpha \alpha}\right)\left(\operatorname{Im} S^{\alpha \alpha}\right) .
$$

This expression agrees with the one given in [60] just by identifying $\operatorname{Re} S^{\alpha \alpha}=-\lambda_{S}^{\ell}$ and $\operatorname{Im} S^{\alpha \alpha}=\lambda_{P}^{\ell}$. Notice that eqs. (4.36) and (4.38) are both exact results for the diagonal contributions to the dipole moments.

\section{Phenomenological discussion}

After deriving analytical expressions for several leptonic observables of interest we now discuss their associated phenomenology.

\subsection{Searches for $\ell_{\alpha} \rightarrow \ell_{\beta} \phi$}

Several searches for $\ell_{\alpha} \rightarrow \ell_{\beta} \phi$ have been performed and used to set experimental contraints on the off-diagonal $S_{A}^{\beta \alpha}$ effective couplings. Let us start with muon decays. The strongest limit on the branching ratio for the 2-body decay $\mu^{+} \rightarrow e^{+} \phi$ was obtained at TRIUMF, finding $\operatorname{BR}(\mu \rightarrow e \phi)<2.6 \times 10^{-6}$ at $90 \%$ C.L. [61]. However, as explained in [55], this experimental limit must be applied with care to the general scenario considered here. The reason is that the experimental setup in [61] uses a muon beam that is highly polarized in the direction opposite to the muon momentum and concentrates the search in the forward region. This reduces the background from the SM process $\mu^{+} \rightarrow e^{+} \nu_{e} \bar{\nu}_{\mu}$, which is strongly suppressed in this region, but also reduces the $\mu^{+} \rightarrow e^{+} \phi$ signal unless the $\phi-e-\mu$ coupling is purely right-handed. Therefore, we obtain a limit valid only when $S_{L}^{e \mu}=0$ :

$$
S_{L}^{e \mu}=0 \quad \Rightarrow \quad\left|S_{R}^{e \mu}\right|<2.7 \times 10^{-11}
$$

A more general limit can also be derived from [61]. Using the spin processed data shown in figure(7) of [61], the authors of [55] obtained the conservative bound $\mathrm{BR}(\mu \rightarrow e \phi) \lesssim 10^{-5}$, valid for any chiral structure of the $S_{A}^{e \mu}$ couplings. This bound is similar to the more recent 
limit obtained by the TWIST collaboration [62], also in the $\sim 10^{-5}$ ballpark. With this value, one finds an upper limit on the $e-\mu$ flavor violating couplings of ${ }^{5}$

$$
\left|S^{e \mu}\right|<5.3 \times 10^{-11},
$$

where we have defined the convenient combination

$$
\left|S^{\beta \alpha}\right|=\left(\left|S_{L}^{\beta \alpha}\right|^{2}+\left|S_{R}^{\beta \alpha}\right|^{2}\right)^{1 / 2}
$$

Several strategies can be followed for newer $\mu \rightarrow e \phi$ searches. The authors of [39] advocate for a new phase of the MEG-II experiment, reconfigured to search for $\mu \rightarrow e \phi$ by placing a Lyso calorimeter in the forward direction. Also, as pointed out in [63, 64] and recently discussed in [39] as well, the limit in eq. (5.2) can be substantially improved by the Mu3e experiment by looking for a bump in the continuous Michel spectrum. The detailed analysis in [64] shows that $\mu \rightarrow e \phi$ branching ratios above $7.3 \times 10^{-8}$ can be ruled out at $90 \%$ C.L.. This would imply a sensitivity to an $\left|S^{e \mu}\right|$ effective coupling as low as $4.5 \times 10^{-12}$, improving an order of magnitude with respect to the limit in eq. (5.2).

Turning to $\tau$ decays, the currently best experimental limits were set by the ARGUS collaboration [65], which found

$$
\begin{aligned}
& \frac{\mathrm{BR}(\tau \rightarrow e \phi)}{\operatorname{BR}(\tau \rightarrow e \nu \bar{\nu})}<0.015, \\
& \frac{\operatorname{BR}(\tau \rightarrow \mu \phi)}{\operatorname{BR}(\tau \rightarrow \mu \nu \bar{\nu})}<0.026,
\end{aligned}
$$

at $95 \%$ C.L. . These limits are weaker than those for muon decays, but still lead to stringent constraints on the LFV $\tau$ couplings with the scalar $\phi$. It is straightforward to find

$$
\begin{aligned}
& \left|S^{e \tau}\right|<5.9 \times 10^{-7}, \\
& \left|S^{\mu \tau}\right|<7.6 \times 10^{-7} .
\end{aligned}
$$

These limits for the LFV couplings to $\tau$ leptons are expected to be improved at Belle II. In fact, new methods for $\tau \rightarrow \ell \phi$ searches at this experiment have been recently proposed [66].

\section{$5.2 \ell_{\alpha} \rightarrow \ell_{\beta} \gamma \phi$ at the MEG experiment}

In order to illustrate the calculation of the phase space integral for a specific case, let us focus on the $\mu \rightarrow e \gamma \phi$ decay and consider the MEG experiment [67]. This experiment has been designed to search for $\mu \rightarrow e \gamma$ and therefore concentrates on $E_{e} \simeq m_{\mu} / 2$ and $\cos \theta_{e \gamma} \simeq-1$ (positron and photon emitted back to back). However, due to the finite experimental resolution, these cuts cannot be imposed with full precision, which makes MEG also sensitive to $\mu \rightarrow e \gamma \phi$. The final MEG results were obtained with the cuts [67]

$$
\cos \theta_{e \gamma}<-0.99963, \quad 51.0<E_{\gamma}<55.5 \mathrm{MeV}, \quad 52.4<E_{e}<55.0 \mathrm{MeV} .
$$

\footnotetext{
${ }^{5}$ See also the recent [39] for a comprehensive discussion of the experimental limit of [61] and how this gets altered for different chiral structures of the $S_{A}^{e \mu}$ couplings.
} 


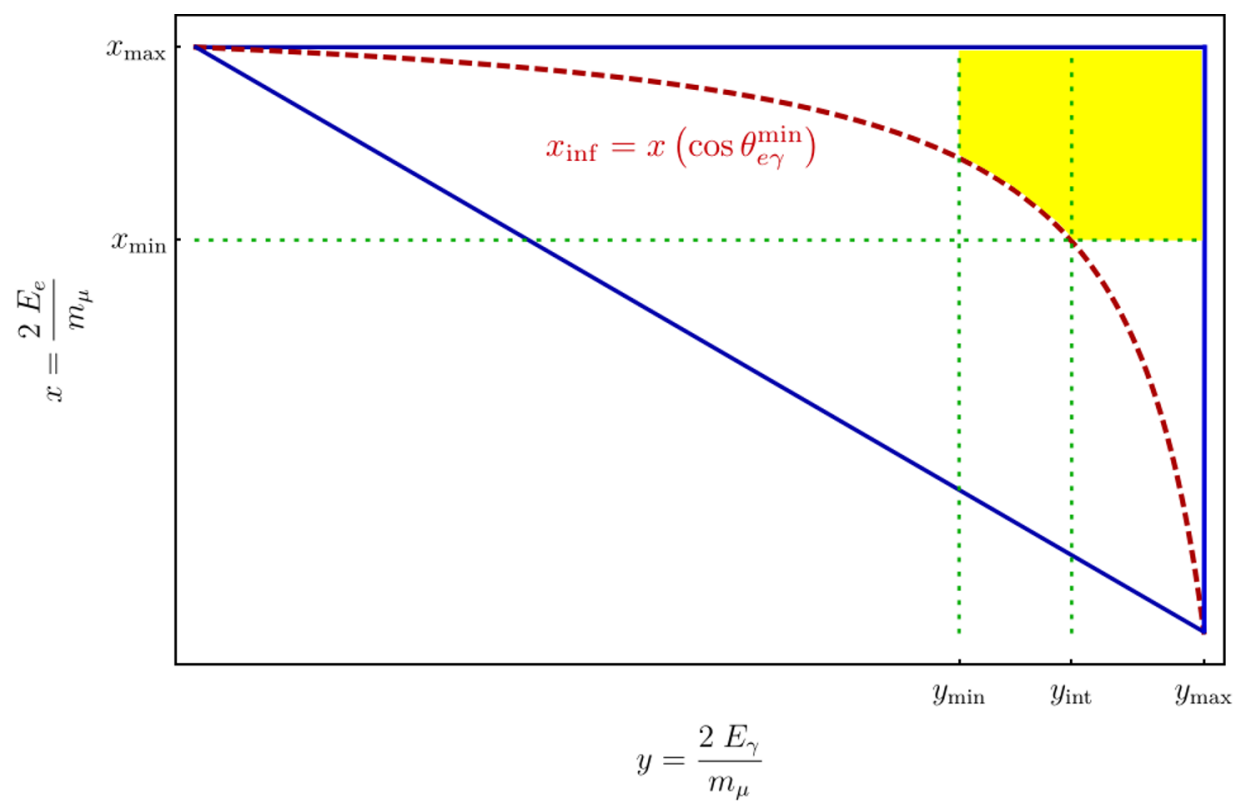

Figure 7. Illustration of the allowed phase space region for the process $\mu \rightarrow e \gamma \phi$ in a given experiment. The blue continuous lines correspond to $\cos \theta_{e \gamma}= \pm 1$ and therefore delimit the total phase space that would be in principle available due to kinematics. The red dashed line represents $x_{\text {inf }}(y)$ and corresponds to the minimal $\theta_{e \gamma}$ angle measurable by the experiment, excluding the region below it. The green dotted straight lines at $x_{\min }$ and $y_{\min }$ are the minimal positron and photon energy, respectively, that the experiment can measure, while $y_{\text {int }}$ is the value of $y$ for which $x_{\min }$ and $x_{\text {inf }}$ intersect. Finally, the yellow surface is the region where we must integrate.

This defines the MEG kinematical region for the calculation of the phase space integral in eq. (4.3) since $\mu \rightarrow e \gamma \phi$ events that fall in this region can be detected by the experiment. For instance, events with $\cos \theta_{e \gamma}<-0.99963$, or equivalently $\theta_{e \gamma}>\theta_{e \gamma}^{\min }=178.441^{\circ}$, were at the reach of MEG. The kinematical region can be divided into two subregions:

$$
\begin{aligned}
y_{\min }=\frac{2 E_{\gamma}^{\min }}{m_{\mu}} & <y<y_{\text {int }}, \\
& x_{\mathrm{inf}}<x<x_{\max }=1,
\end{aligned}
$$

and

$$
\begin{aligned}
y_{\text {int }} & <y<y_{\max }=1, \\
x_{\min }=\frac{2 E_{e}^{\min }}{m_{\mu}} & <x<x_{\max },
\end{aligned}
$$

where $x_{\text {inf }}=x_{\text {inf }}(y)$ is the value of $x$ such that $\cos \theta_{e \gamma}=\cos \theta_{e \gamma}^{\min }$ for each value of $y$. This can be easily found by solving eq. (4.5):

$$
x_{\mathrm{inf}}=\frac{2(1-y)}{2-y\left(1-\cos \theta_{e \gamma}^{\min }\right)} .
$$

Finally $y_{\text {int }}$ is the value of $y$ for which $x_{\min }$ and $x_{\text {inf }}$ coincide. These two subregions are illustrated in figure 7 , where the experimental restrictions have been modified for the sake of 

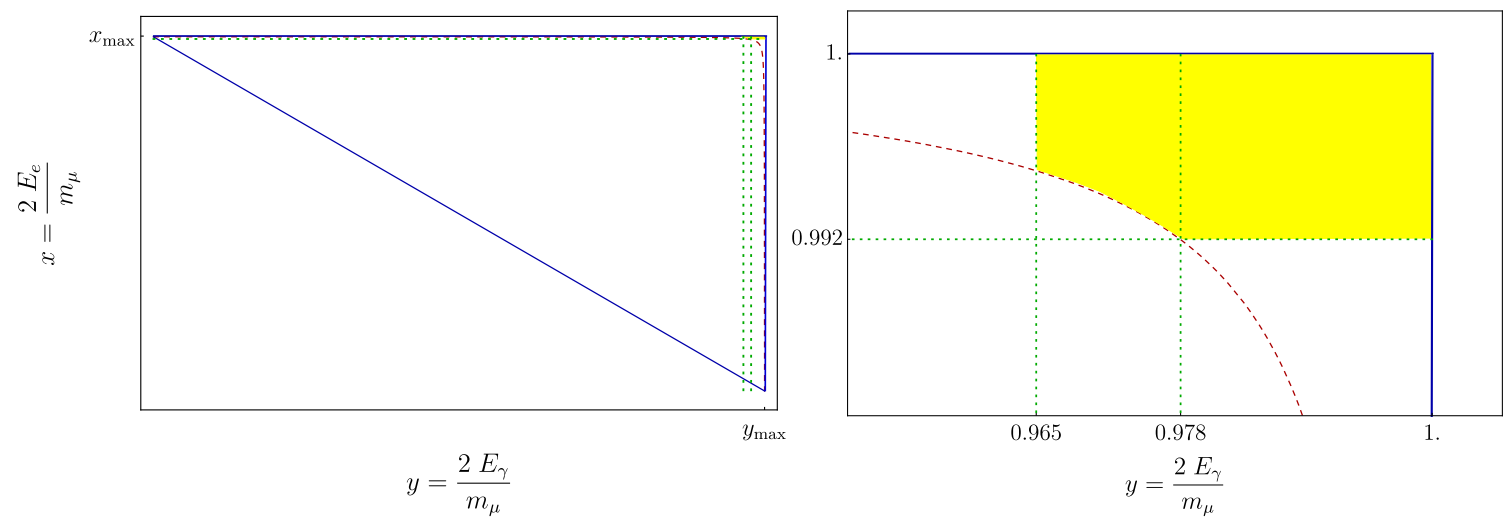

Figure 8. Realistic version of the phase space region limited by the experimental cuts of the MEG experiment, given in eq. (5.6). The figure on the right shows a zoom of the figure on the left, centered on the colored surface.

clarity by enlarging the kinematical region of interest. A realistic representation obtained with the MEG cuts in eq. (5.6) is shown in figure 8. This clearly illustrates the strong suppression due to the phase space integral.

Having explained how to compute the phase space integral and illustrated the strong suppression it introduces, we can obtain results for the MEG experiment. Using the cuts in eq. (5.6), the phase space integral in eq. (4.3) can be numerically computed to find

$$
\mathcal{I}\left(x_{\min }, y_{\text {min }}\right)_{\mathrm{MEG}}=3.8 \times 10^{-8} .
$$

Combining this result with eq. (4.2), we obtain the branching ratio of $\mu \rightarrow e \gamma \phi$ restricted to the MEG phase space, obtaining

$$
\operatorname{BR}_{\mathrm{MEG}}(\mu \rightarrow e \gamma \phi)=1.5 \times 10^{5}\left(\left|S_{L}^{e \mu}\right|^{2}+\left|S_{R}^{e \mu}\right|^{2}\right) .
$$

MEG results require $\mathrm{BR}(\mu \rightarrow e \gamma)<4.2 \times 10^{-13}$ [67], a bound that must also be satisfied by $\operatorname{BR}_{\mathrm{MEG}}(\mu \rightarrow e \gamma \phi)$. This leads to

$$
\left|S^{e \mu}\right|<1.6 \times 10^{-9} .
$$

This bound is notably worse than the one given in eq. (5.2), as expected due to the strong phase space suppression at MEG, an experiment that is clearly not designed to search for $\mu \rightarrow e \gamma \phi$.

More stringent bounds were obtained at the Crystal Box experiment at LAMPF [6870]. Several searches were performed, with different experimental cuts and branching ratio bounds. These result in different limits on the $\left|S^{e \mu}\right|$ effective coupling, as shown in table 1 . Adapting the limit from the $\mu \rightarrow e \gamma$ search in [68] along the lines followed in the previous discussion for MEG, we find

$$
\left|S^{e \mu}\right|<9.5 \times 10^{-11} .
$$

This bound is still not better than the one given in eq. (5.2), but it is in the same ballpark. A very similar bound is obtained with the results of a later analysis, in this case more specific to $\mu \rightarrow e \gamma \phi[69,70]$. 


\begin{tabular}{|ccccccc|}
\hline References & $\theta_{e \gamma}^{\min }$ & $E_{\gamma}^{\min }[\mathrm{MeV}]$ & $E_{e}^{\min }[\mathrm{MeV}]$ & $\mathcal{I}\left(x_{\min }, y_{\min }\right)$ & BR bound & Limit on $\left|S^{e \mu}\right|$ \\
\hline$[68]$ & $160^{\circ}$ & 40 & 44 & $1.3 \times 10^{-3}$ & $4.9 \times 10^{-11}$ & $9.5 \times 10^{-11}$ \\
{$[69,70]$} & $140^{\circ}$ & 38 & 38 & $1.1 \times 10^{-2}$ & $1.1 \times 10^{-9}$ & $1.6 \times 10^{-10}$ \\
\hline
\end{tabular}

Table 1. Results in the search for $\mu \rightarrow e \gamma \phi$ at the Crystal Box experiment.

Finally, the Mu3e experiment is not well equipped to detect the photon in $\mu \rightarrow e \gamma \phi$ and therefore cannot improve on these limits. As explained in [64], a future Muзe-Gamma experiment including a photon conversion layer could increase the sensitivity to $\mu \rightarrow e \gamma \phi$.

\section{$5.3 \quad \ell_{\alpha} \rightarrow \ell_{\beta} \gamma$ vs. $\ell_{\alpha} \rightarrow \ell_{\beta} \ell_{\beta} \ell_{\beta}$}

The LFV decays $\ell_{\alpha} \rightarrow \ell_{\beta} \gamma$ and $\ell_{\alpha} \rightarrow \ell_{\beta} \ell_{\beta} \ell_{\beta}$ constitute complementary probes of the underlying physics. While $\ell_{\alpha} \rightarrow \ell_{\beta} \gamma$ only receives contributions from dipole operators, $\ell_{\alpha} \rightarrow \ell_{\beta} \ell_{\beta} \ell_{\beta}$ is induced by dipole as well as non-dipole operators. Their relative importance can be studied by means of the ratio

$$
R_{\alpha \beta}=\frac{\operatorname{BR}\left(\ell_{\alpha} \rightarrow \ell_{\beta} \ell_{\beta} \ell_{\beta}\right)}{\operatorname{BR}\left(\ell_{\alpha} \rightarrow \ell_{\beta} \gamma\right)}
$$

In models in which the $\ell_{\alpha} \rightarrow \ell_{\beta} \ell_{\beta} \ell_{\beta}$ amplitude is clearly dominated by dipole contributions, the two branching ratios are strongly correlated and one can make a definite prediction for $R_{\mu e}$. In fact, since $\ell_{\alpha} \rightarrow \ell_{\beta} \ell_{\beta} \ell_{\beta}$ involves an additional electromagnetic coupling constant, one expects $R_{\alpha \beta} \ll 1$. Departures from this prediction would clearly point towards a nondipole dominant contribution. We now consider this issue in the presence of an ultralight scalar, which contributes at tree-level to $\ell_{\alpha} \rightarrow \ell_{\beta} \ell_{\beta} \ell_{\beta}$ via scalar (and hence non-dipole) operators. Contrary to the above-mentioned dipole-dominated scenarios, in this case one generally expects $R_{\alpha \beta} \gg 1$, as shown below.

However, before we move on to the discussion of the interplay between $\ell_{\alpha} \rightarrow \ell_{\beta} \gamma$ and $\ell_{\alpha} \rightarrow \ell_{\beta} \ell_{\beta} \ell_{\beta}$, we would like to point out that light scalars may offer additional experimental handles in $\ell_{\alpha} \rightarrow \ell_{\beta} \ell_{\beta} \ell_{\beta}$. In particular, the authors of [71] showed that a light scalar produced on-shell in $\ell_{\alpha}^{-} \rightarrow \ell_{\beta}^{-} \phi$ that later decays as $\phi \rightarrow \ell_{\beta}^{-} \ell_{\beta}^{+}$may lead to observable displaced vertices. This interesting possibility is, however, not possible in the ultralight scalar scenario considered here.

General dipole contributions. First, we consider the general case of a scenario in which dipole contributions are independent of the non-dipole ones induced by the ultralight scalar $\phi$. This would be the case of a model containing additional LFV sources, not related to $\phi$. In order to evaluate the relevance of the new contributions to $\ell_{\alpha} \rightarrow \ell_{\beta} \ell_{\beta} \ell_{\beta}$ mediated by the scalar $\phi$ we drop the 4 -fermion operators in eq. (2.4) and consider a simplified effective Lagrangian containing only left-handed photonic dipole and scalar-mediated operators

$$
\mathcal{L}_{\mathrm{LFV}}^{\mathrm{simp}}=\frac{e m_{\alpha}\left(K_{2}^{L}\right)^{\beta \alpha}}{2} \bar{\ell}_{\beta} \sigma^{\mu \nu} P_{L} \ell_{\alpha} F_{\mu \nu}+S_{L}^{\beta \alpha} \phi \bar{\ell}_{\beta} P_{L} \ell_{\alpha}+\text { h.c. } .
$$




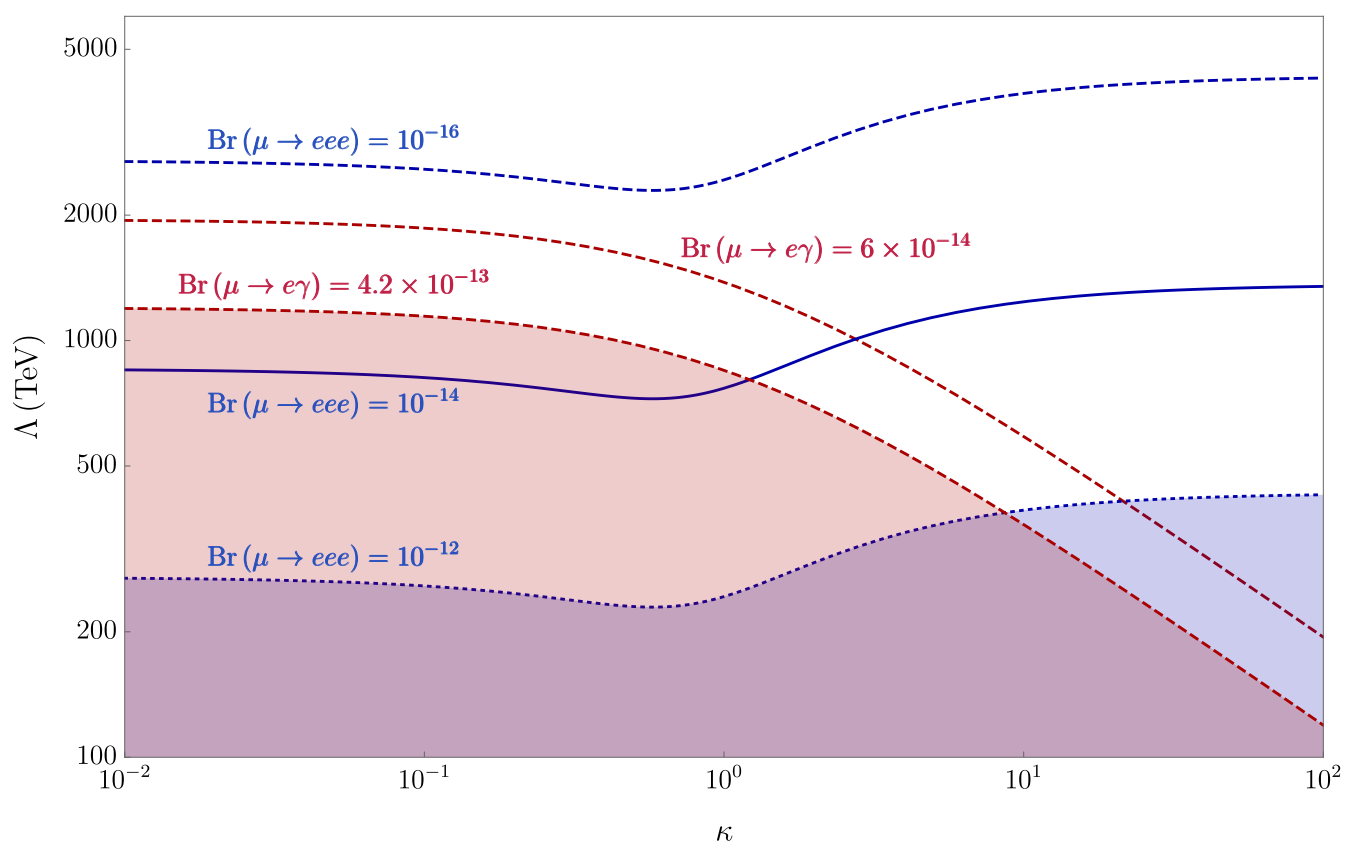

Figure 9. Contours of $\mathrm{BR}(\mu \rightarrow e \gamma)$ and $\mathrm{BR}(\mu \rightarrow e e e)$ in the $\kappa-\Lambda$ plane. The lowest values correspond to the future sensitivities for the MEG-II and Mu3e experiments, while colored regions are excluded due to the current bounds $\operatorname{BR}(\mu \rightarrow e \gamma)<4.2 \cdot 10^{-13}$ and $\operatorname{BR}(\mu \rightarrow e e e)<10^{-12}[73]$. These results have been obtained with the effective Lagrangian in eq. (2.1) and the parametrization in eq. (5.16).

Then, inspired by [72], we parametrize the $K_{2}^{L}$ and $S_{L}$ coefficients as

$$
e\left(K_{2}^{L}\right)^{\beta \alpha} \equiv \frac{1}{(\kappa+1) \Lambda^{2}}, \quad S_{L}^{\beta \alpha} \equiv m_{\alpha} \frac{\kappa}{(\kappa+1) \Lambda} .
$$

$\Lambda$ is a dimensionful parameter that represents the energy scale at which these coefficients are induced, while $\kappa$ is a dimensionless parameter that accounts for the relative intensity of these two interactions. ${ }^{6}$ In case of $\kappa \ll 1$, the dipole operator dominates, while the scalar mediated contribution dominates for $\kappa \gg 1$. We point out that $m_{\alpha}$ in eqs. (5.15) and (5.16) is a global factor given by the mass of the heaviest charged lepton in the process and that eq. (5.16) assumes $S_{L}^{\beta \alpha}=S_{L}^{\beta \beta}$.

Figure 9 shows $\mathrm{BR}(\mu \rightarrow e \gamma)$ and $\mathrm{BR}(\mu \rightarrow e e e)$ as a function of $\Lambda$ and $\kappa$. Our results are compared to the current bounds and the future sensitivities for the MEG-II and Mu3e experiments. We observe that for $\kappa \gg 1$ and $\operatorname{BR}(\mu \rightarrow e e e)>10^{-16}, \Lambda$ must be necessarily below $\sim 3000 \mathrm{TeV}$. A slightly lower upper limit for $\Lambda$ is found when $\kappa \ll 1$ and $\operatorname{BR}(\mu \rightarrow$ $e \gamma)>10^{-14}$. These are precisely the final expected sensitivities in MEG-II and Mu3e. Furthermore, we note that the search for the scalar mediated contribution in Mu3e will actually be very constraining in all the parameter space. Similar results are shown for

\footnotetext{
${ }^{6}$ We normalize $S_{L}$ by introducing the mass of the heaviest charged lepton involved in each process. However, this is done only for the purpose of this analysis. In the rest of the paper we do not assume any hierarchy among the couplings proportional to the charged lepton masses.
} 

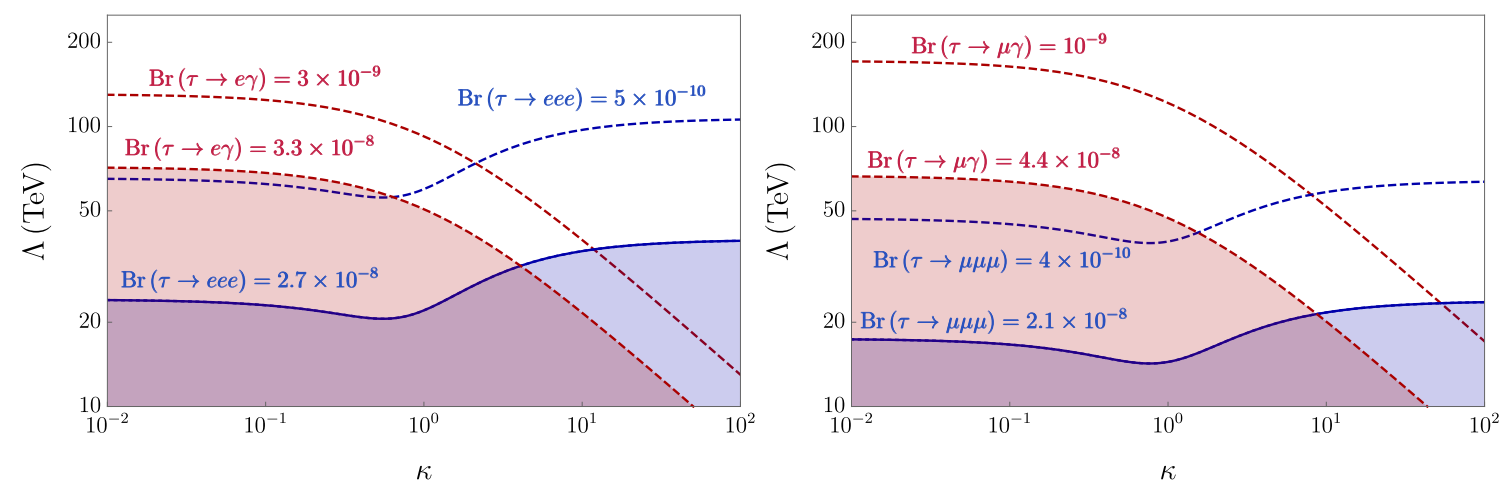

Figure 10. Contours of $\operatorname{BR}(\tau \rightarrow e \gamma)$ and $\operatorname{BR}(\tau \rightarrow e e e)$, on the left, and $\operatorname{BR}(\tau \rightarrow \mu \gamma)$ and $\operatorname{BR}(\tau \rightarrow \mu \mu \mu)$, on the right, in the $\kappa-\Lambda$ plane. The lowest values correspond to the expected future sensitivities of the Belle II experiment [6], while colored regions are excluded due to the current bounds $\operatorname{BR}(\tau \rightarrow e \gamma)<3.3 \cdot 10^{-8}, \operatorname{BR}(\tau \rightarrow \mu \gamma)<4.4 \cdot 10^{-8}, \operatorname{BR}(\tau \rightarrow e e e)<2.7 \cdot 10^{-8}$ and $\operatorname{BR}(\tau \rightarrow \mu \mu \mu)<2.1 \cdot 10^{-8}[73]$. These results have been obtained with the effective Lagrangian in eq. (2.1) and the parametrization in eq. (5.16).

$\tau$ decays in figure 10. In this case, the current experimental limits are expected to be improved by about one order of magnitude by the $\mathrm{LHCb}$ and Belle II collaborations, which will search for the $\tau \rightarrow \ell_{\beta} \gamma$ and $\tau \rightarrow \ell_{\beta} \ell_{\beta} \ell_{\beta}$ decays, with $\ell_{\beta}=e, \mu$. This figure has been obtained using the expected sensitivities by the Belle II experiment presented in [6]. We find that for low values of $\kappa$, i.e. $\kappa \ll 1$, the current limit on $\operatorname{BR}(\tau \rightarrow e \gamma)$ implies the non-observation of $\tau \rightarrow$ eee at Belle II. This would therefore require a larger value of $\kappa$, to enhance the relative weight of the 3-body decay. Qualitatively similar results are obtained for $\tau \rightarrow \mu$ transitions.

$\phi$-induced dipole contributions. We now consider the generation of dipole operators by loops involving the ultralight scalar $\phi$, as discussed in section 4.3 and shown in figure 2 . In this scenario, we assume that $\phi$ provides the dominant (or, of course, only) contribution to dipole operators. For the sake of simplicity, the couplings $S^{e e}$ and $S_{L, R}^{e \mu}$ will be the only ones allowed to be different from zero in the analysis that follows. They will also be taken to be real. In this case, the general expressions for $K_{2}^{L}$ and $K_{2}^{R}$ given in eqs. (4.8) and (4.9) lead to

$$
\begin{aligned}
\left(K_{2}^{L}\right)^{e \mu}= & \frac{S^{e e}}{96 \pi^{2} m_{\mu}^{3}}\left\{3 m_{\mu} S_{R}^{e \mu}+m_{e}\left(-6 S_{L}^{e \mu}+2 \pi^{2} S_{L}^{e \mu}+3 S_{R}^{e \mu}\right)\right. \\
& \left.+3 m_{e} S_{L}^{e \mu} \log \left(-\frac{m_{e}^{2}}{m_{\mu}^{2}}\right)\left[1+\log \left(-\frac{m_{e}^{2}}{m_{\mu}^{2}}\right)\right]\right\}, \\
\left(K_{2}^{R}\right)^{e \mu}= & \frac{S^{e e}}{96 \pi^{2} m_{\mu}^{3}}\left\{3 m_{\mu} S_{L}^{e \mu}+m_{e}\left(-6 S_{R}^{e \mu}+2 \pi^{2} S_{R}^{e \mu}+3 S_{L}^{e \mu}\right)\right. \\
& \left.+3 m_{e} S_{R}^{e \mu} \log \left(-\frac{m_{e}^{2}}{m_{\mu}^{2}}\right)\left[1+\log \left(-\frac{m_{e}^{2}}{m_{\mu}^{2}}\right)\right]\right\},
\end{aligned}
$$


where we have expanded at first order in $m_{e}$. These expressions allow us to compute the $R_{\mu e}$ ratio defined in eq. (5.14). Defining the mass ratio $r=\frac{m_{\mu}^{2}}{m_{e}^{2}}$, we do that for some simplified scenarios:

- Scenario 1: $S_{L}^{e \mu}=0$ or $S_{R}^{e \mu}=0$

$$
R_{\mu e}^{(1)} \approx \frac{4 \pi r}{3 \alpha} \frac{12 \log r-53}{|\log (-r)|^{4}+r} \approx 3.2 \cdot 10^{4}
$$

- Scenario 2: $S_{L}^{e \mu}=S_{R}^{e \mu}$

$$
R_{\mu e}^{(2)} \approx \frac{4 \pi r}{3 \alpha} \frac{12 \log r-53}{\left|\log ^{2}(-r)+\sqrt{r}\right|} \approx 1.9 \cdot 10^{4} .
$$

- Scenario 3: $S_{L}^{e \mu}=-S_{R}^{e \mu}$

$$
R_{\mu e}^{(3)} \approx \frac{4 \pi r}{3 \alpha} \frac{12 \log r-53}{\left|\log ^{2}(-r)-\sqrt{r}\right|} \approx 1.1 \cdot 10^{5} .
$$

We find that $R_{\mu e} \gg 1$ in these scenarios. This, however, was expected, since $\ell_{\alpha} \rightarrow$ $\ell_{\beta} \ell_{\beta} \ell_{\beta}$ is induced at tree-level by $\phi$ exchange, while $\ell_{\alpha} \rightarrow \ell_{\beta} \gamma$ can only take place at loop order. More interestingly, different scenarios for the $\phi$ couplings lead to very different predictions for $R_{\mu e}$. This would in principle allow us to determine the nature of the scalar $\phi$ if positive signals are observed for both $\mu \rightarrow e \gamma$ and $\mu \rightarrow e e e$, and both branching ratios can be experimentally determined.

\subsection{Lepton magnetic and electric dipole moments}

At present, there is a discrepancy between the experimental determination of the electron and muon AMMs and their SM predicted values [74-80]

$$
\begin{aligned}
\Delta a_{e} & =a_{e}^{\exp }-a_{e}^{\mathrm{SM}}=(-87 \pm 36) \times 10^{-14} \\
\Delta a_{\mu} & =a_{\mu}^{\exp }-a_{\mu}^{\mathrm{SM}}=(27.1 \pm 7.3) \times 10^{-10}
\end{aligned}
$$

where

$$
a_{\beta}=\frac{g_{\beta}-2}{2} .
$$

In the case of the muon anomalous magnetic moment, the deviation is at the level of $\sim 4 \sigma$, whereas for the electron anomalous magnetic moment the significance is a little lower, slightly below $\sim 3 \sigma .^{7}$ While further measurements (and possibly improved theoretical calculations) are required to fully confirm these anomalies, these intriguing deviations can

\footnotetext{
${ }^{7}$ See also the very recent calculation of the hadronic vacuum polarization contribution by the BudapestMarseilles-Wuppertal collaboration [81], which brings the SM prediction for the muon anomalous magnetic moment into agreement with the experimental measurement. However, this result seems to lead to tension with electroweak data [82].
} 
be interpreted as a possible hint of new physics [83]. In particular, the sign difference between $\Delta a_{e}$ and $\Delta a_{\mu}$ and the relatively large value of $\left|\Delta a_{e}\right|$ may indicate the presence of new physics contributions that do not scale with the square of the corresponding charged lepton masses [84]. In what concerns the EDMs of the charged leptons, the SM predicts tiny values, well beyond the experimental prospects in the near future. Therefore, any measurement of a non-zero charged lepton EDM would be a clear indication of CP-violating new physics effects. The current best limits for the electron and muon EDMs are $[85,86]$

$$
\begin{aligned}
& \left|d_{e}\right|<1.1 \times 10^{-29} e \mathrm{~cm}, \\
& \left|d_{\mu}\right|<1.5 \times 10^{-19} e \mathrm{~cm},
\end{aligned}
$$

both at $95 \%$ C.L. .

Figure 11 shows favored regions for the diagonal coupling $S^{e e}$ due to the electron AMM and EDM. As shown on the left panel, the bound on the electron EDM strongly constrains the $S^{e e}$ coupling, which must be essentially purely real or essentially purely imaginary. However, one can find regions in the parameter space that explain the $(g-2)_{e}$ anomaly, compatible with the bound on the electron EDM. Given the low significance of the $(g-2)_{e}$ anomaly, one stays within the $3 \sigma$ region even if $S^{e e}=0$, but if $\operatorname{Re} S^{e e} \lesssim 10^{-13}$, a value of about $\operatorname{Im} S^{e e} \sim 10^{-5}$ would actually achieve agreement at the $1 \sigma$ level. The deviation in $(g-2)_{\mu}$ is more significant, and this implies that one must introduce larger $S^{\mu \mu}$ values in order to reconcile the theoretical prediction with the experimental measurement. This is shown on figure 12. In this case, the bound from the muon EDM does not impose strong restrictions on the parameter space, as can be clearly seen in the left panel. However, larger $S^{\mu \mu}$ couplings, of the order of $10^{-4}$, are necessary in order to explain the current deviation between theory and experiment. In both cases, the required values for $S^{e e}$ and $S^{\mu \mu}$ are in conflict with the bounds discussed in section 3, see eqs. (3.2) and (3.3), and therefore a mechanism to suppress the processes from which they are derived would be necessary for the ultralight scalar $\phi$ to be able to provide an explanation to the current $g-2$ anomalies.

Finally, we have explored whether the electron and muon AMM anomalies can be explained by purely off-diagonal contributions. In the following we consider vanishing diagonal couplings and real non-zero off-diagonal couplings. In this scenario the contribution to the charged leptons EDMs vanish and the AMMs strongly correlate with LFV observables. In fact, the bounds derived in section 4.7 from the non-observation of $\ell_{\alpha} \rightarrow \ell_{\beta} \phi$ imply that an explanation to the observed deviations cannot be achieved. In particular, we find that $S_{L}^{e \mu} \sim-S_{R}^{e \mu} \sim 2 \times 10^{-4}$ or $S_{L}^{e \tau} \sim-S_{R}^{e \tau} \sim 7 \times 10^{-4}$ are needed in order to explain the $(g-2)_{e}$ deviation. Regarding the muon AMM anomaly, only with the $\mu-\tau-\phi$ coupling one can obtain a positive contribution, requiring $S_{L}^{\mu \tau} \sim S_{R}^{\mu \tau} \sim 3 \times 10^{-3}$ to explain the $(g-2)_{\mu}$ deviation. In all cases, the required off-diagonal couplings are several orders of magnitude larger than the limits in eqs. (5.2) and (5.5). We therefore conclude that the explanation of the electron and muon AMMs anomalies must come from diagonal contributions, whereas the off-diagonal ones can only play a subdominant role. 

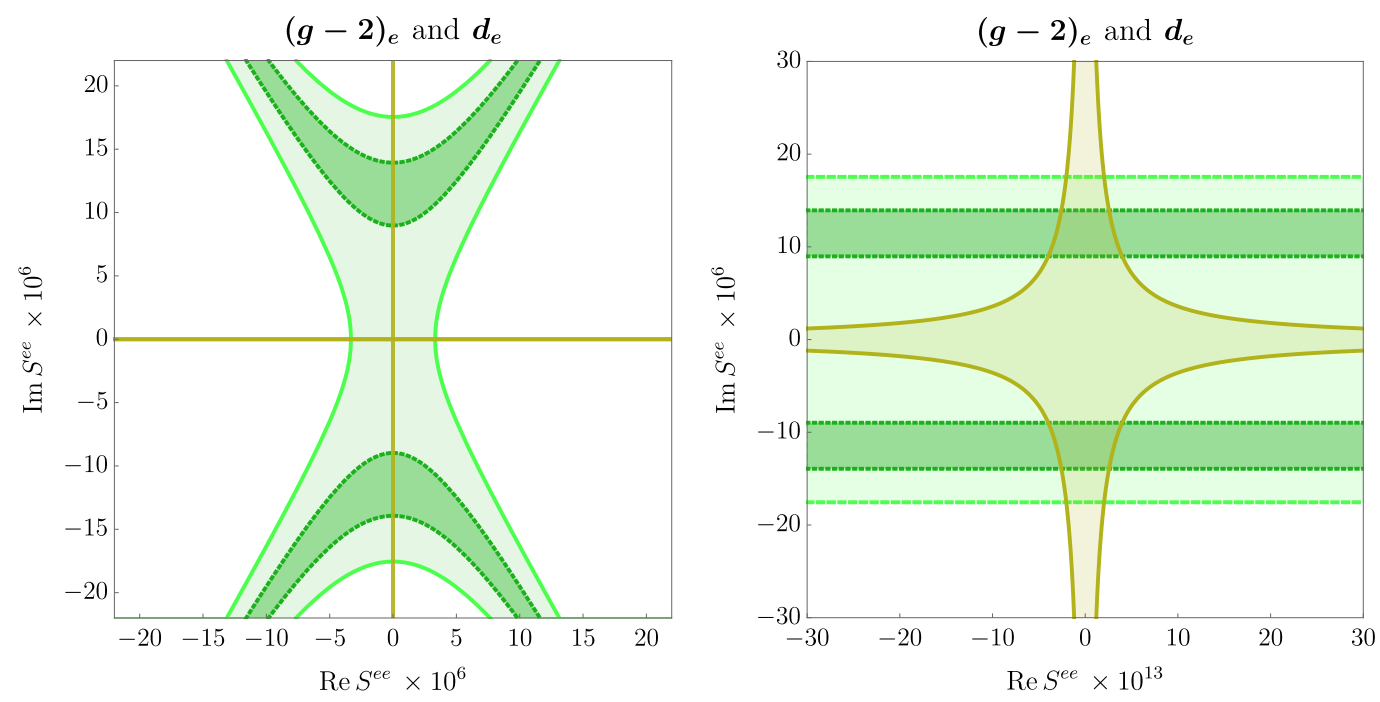

Figure 11. Favored region for the diagonal coupling $S^{e e}$, due to the electron anomalous magnetic and electric dipole moments. Within the light (dark) green region, the deviation in the electron AMM is explained at the $3 \sigma(1 \sigma)$ level. The region delimited by the orange continuous lines is the parameter space allowed by the current experimental upper bound of the electron EDM. In the figure on the right, the abscissa axis has been zoomed.
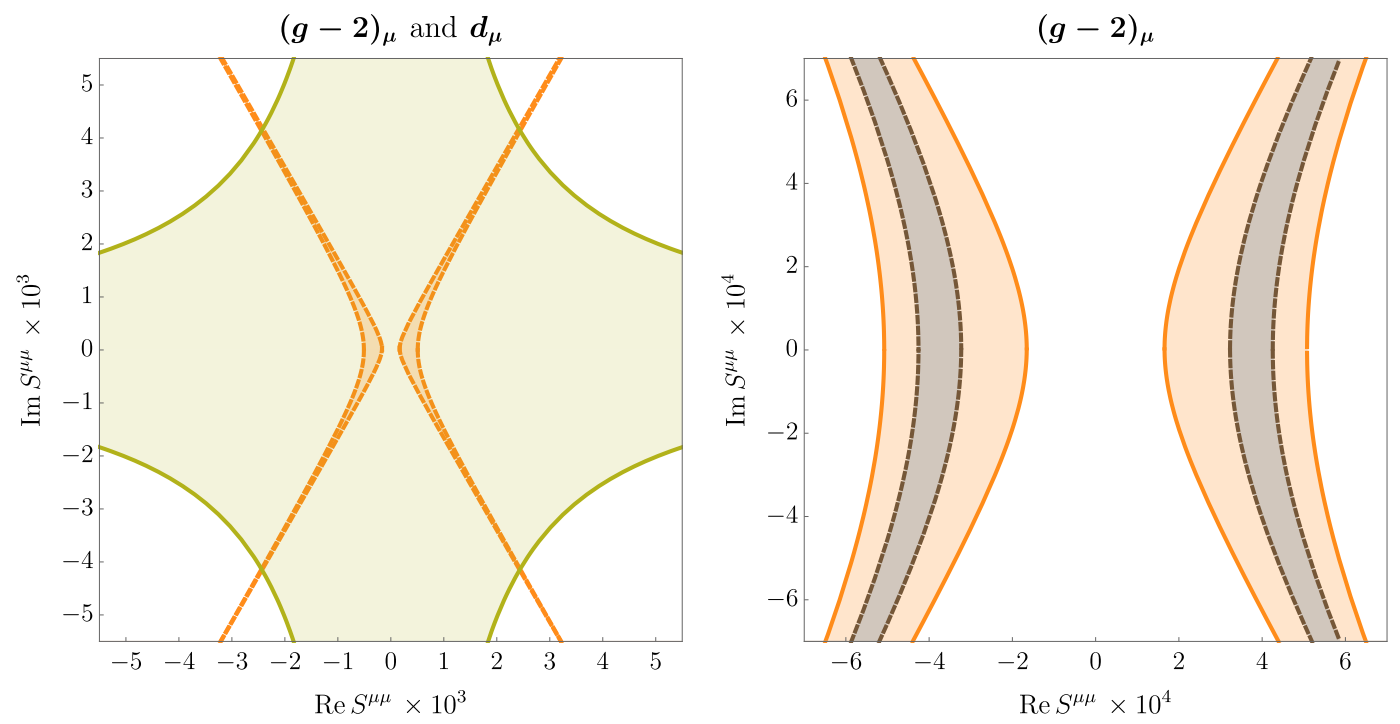

Figure 12. Favored regions for the diagonal coupling $S^{\mu \mu}$, due to the muon anomalous magnetic and electric dipole moments. In the figure on the left it is seen that the bound from the muon EDM (yellow continuous curves) does not restrict too much the AMM of the muon (orange dashed curves). On the right figure, only the muon AMM is represented and within the light (dark) region, the current experimental deviation is explained at the $3 \sigma(1 \sigma)$ level. 


\section{Conclusions}

Ultralight scalars appear in a wide variety of SM extensions, either as very light states or as exactly massless Goldstone bosons. Examples include the axion and the majoron, two well-motivated hypothetical particles at the core of two fundamental problems: the conservation of $\mathrm{CP}$ in the strong interactions and the origin of neutrino masses. These states, as well as other ultralight scalars, can be produced in many leptonic processes or act as their mediators, leading to many exotic signatures.

In this work we have explored the impact of ultralight scalars in many leptonic observables. We have adopted a model independent general approach, taking into account both scalar and pseudoscalar interactions to charged leptons, therefore going beyond most existing studies. First, we have briefly reviewed the current bounds from stellar cooling, which set important constraints on the diagonal couplings, and discussed indirect limits from the 1-loop generation of a coupling to photons. Then, we have obtained analytical expressions for a wide variety of leptonic observables. We have revisited the decays $\ell_{\alpha} \rightarrow \ell_{\beta} \phi$ and $\ell_{\alpha} \rightarrow \ell_{\beta} \gamma \phi$, in which the scalar $\phi$ is produced, and provided complete expressions for the radiative LFV decays $\ell_{\alpha} \rightarrow \ell_{\beta} \gamma$, as well as for the 3-body decays $\ell_{\alpha}^{-} \rightarrow \ell_{\beta}^{-} \ell_{\beta}^{-} \ell_{\beta}^{+}, \ell_{\alpha}^{-} \rightarrow \ell_{\beta}^{-} \ell_{\gamma}^{-} \ell_{\gamma}^{+}$and $\ell_{\alpha}^{-} \rightarrow \ell_{\beta}^{+} \ell_{\gamma}^{-} \ell_{\gamma}^{-}$, in which $\phi$ contributes as mediator. The effect of ultralight scalars on the charged leptons anomalous magnetic and electric dipole moments has also be discussed. Finally, several phenomenological aspects of this scenario are explored. After deriving limits on off-diagonal couplings from lepton flavor violating observables, we have shown that an explanation to the $(g-2)_{e}$ and $(g-2)_{\mu}$ anomalies is possible in this scenario. We have also shown that the observables discussed in this paper are indeed complementary.

The phenomenology of ultralight scalars is very rich, since they are kinematically accessible in most high- and low-energy processes. We have discussed many purely leptonic processes, but if $\phi$ couples to quarks as well, many hadronic and semi-leptonic channels open. This could give rise to many signatures at kaon factories [87]. Furthermore, ultralight scalars may leave their footprints in other processes. For instance, they can be produced and emitted in tritium beta decay [88] or $\mu-e$ conversion in nuclei [89], have a strong impact in leptogenesis [90], and give rise to non-resonant phenomena at colliders [91]. In our opinion, this diversity of experimental signatures and their potential to unravel some of the most important problems in particle physics through their connection to ultralight scalars merits further investigation. 


\section{Acknowledgments}

The authors are grateful to Julian Heeck, Mario Reig and Martin Hirsch for fruitful discussions. Work supported by the Spanish grants FPA2017-85216-P (MINECO/AEI/FEDER, UE), SEJI/2018/033 (Generalitat Valenciana) and FPA2017-90566-REDC (Red Consolider MultiDark). The work of PE is supported by the FPI grant PRE2018-084599. AV acknowledges financial support from MINECO through the Ramón y Cajal contract RYC2018025795-I.

\section{A Parametrization in terms of derivative interactions}

Eq. (2.1) is completely general and includes both scalar and pseudoscalar interactions of the field $\phi$ with a pair of charged leptons. An alternative parametrization in terms of derivative interactions is given by

$$
\mathcal{L}_{\ell \ell \phi}=\left(\partial_{\mu} \phi\right) \bar{\ell}_{\beta} \gamma^{\mu}\left(\widetilde{S}_{L}^{\beta \alpha} P_{L}+\widetilde{S}_{R}^{\beta \alpha} P_{R}\right) \ell_{\alpha}+\text { h.c. . }
$$

The coefficients $\widetilde{S}_{L, R}$ have dimensions of mass $^{-1}$ and we consider all possible flavor combinations: $\beta \alpha=\{e e, \mu \mu, \tau \tau, e \mu, e \tau, \mu \tau\}$. Notice that the diagonal $\ell_{\beta}-\ell_{\beta}-\phi$ vertex is proportional to $\left(\widetilde{S}_{L}+\widetilde{S}_{L}^{*}\right)^{\beta \beta} P_{L}+\left(\widetilde{S}_{R}+\widetilde{S}_{R}^{*}\right)^{\beta \beta} P_{R}$, and therefore the diagonal couplings can be taken to be real without loss of generality. As will be shown below, eq. (A.1) only includes pseudoscalar interactions for $\phi$. Therefore, it can be thought of as a particularization of eq. $(2.1) .^{8}$

Physical observables must be independent of the parametrization chosen. We proceed to show now that the two parametrizations considered here are completely equivalent for a pure pseudoscalar in processes involving on-shell leptons. First, we recall the equations of motion for the lepton fields $\ell_{\alpha}$ and its conjugate $\bar{\ell}_{\alpha}$

$$
\begin{aligned}
& i \gamma^{\mu} \partial_{\mu} \ell_{\alpha}-m_{\alpha} \ell_{\alpha}=0, \\
& i \partial_{\mu} \bar{\ell}_{\alpha} \gamma^{\mu}+m_{\alpha} \bar{\ell}_{\alpha}=0,
\end{aligned}
$$

valid for on-shell leptons. One can now rewrite eq. (A.1) as the sum of a total derivative and a derivative acting on the lepton fields. The total derivative does not contribute to the action, whereas the derivative on the lepton fields can be replaced using the equations of motion in eq. (A.2). This leads to

$$
\begin{aligned}
\mathcal{L}_{\ell \ell \phi} & =-i \phi \bar{\ell}_{\beta}\left[\left(m_{\beta} \widetilde{S}_{L}^{\beta \alpha}-m_{\alpha} \widetilde{S}_{R}^{\beta \alpha}\right) P_{L}+\left(m_{\beta} \widetilde{S}_{R}^{\beta \alpha}-m_{\alpha} \widetilde{S}_{L}^{\beta \alpha}\right) P_{R}\right] \ell_{\alpha}+\text { h.c. } \\
& \equiv \phi \bar{\ell}_{\beta}\left(S_{L}^{\beta \alpha} P_{L}+S_{R}^{\beta \alpha} P_{R}\right) \ell_{\alpha}+\text { h.c. }
\end{aligned}
$$

\footnotetext{
${ }^{8}$ The parametrization in eq. (A.1) is completely general if $\phi$ is a pure pseudoscalar, usually the case of the Goldstone bosons in many models. In such scenarios, the two parametrizations for the effective Lagrangian $\mathcal{L}_{\ell \ell \phi}$ introduced here are related to two possible ways to parametrize the Goldstone boson. Eq. (2.1) follows from a cartesian parametrization, that splits a complex scalar field in terms of its real and imaginary components. Alternatively, the parametrization in terms of derivative interactions in eq. (A.1) would follow from a polar parametrization, that splits a complex scalar field in terms of its modulus and phase. As we will prove below, they lead to the same results for observables involving on-shell leptons.
} 
Therefore we find a dictionary between the $S_{X}$ and $\widetilde{S}_{X}$ coefficients

$$
\begin{aligned}
S_{L}^{\beta \alpha} & =i\left(m_{\alpha} \widetilde{S}_{R}^{\beta \alpha}-m_{\beta} \widetilde{S}_{L}^{\beta \alpha}\right), \\
S_{R}^{\beta \alpha} & =i\left(m_{\alpha} \widetilde{S}_{L}^{\beta \alpha}-m_{\beta} \widetilde{S}_{R}^{\beta \alpha}\right),
\end{aligned}
$$

which for the diagonal couplings reduces to

$$
S^{\beta \beta}=S_{L}^{\beta \beta}+S_{R}^{\beta \beta *}=2 i m_{\beta}\left(\widetilde{S}_{R}^{\beta \beta}-\widetilde{S}_{L}^{\beta \beta}\right)
$$

Since both $\widetilde{S}_{X}^{\beta \beta}$ are real parameters, eq. (A.6) implies that the diagonal $S^{\beta \beta}$ couplings must be purely imaginary. It is straightforward to show that, in this case, the flavor conserving interactions of $\phi$ in eq. (2.1) are proportional to $\gamma_{5}$ (see eq. (3.1)). This proves that eq. (A.1) is not general, but only includes pseudoscalar interactions, and there is no one-to-one correspondence between the two parametrizations. Given a set of $\widetilde{S}_{X}$ couplings, one can always find the corresponding $S_{X}$ couplings using eqs. (A.4) and (A.5). However, certain sets of $S_{X}$ couplings, namely those with non-vanishing real parts, cannot be expressed in terms of $\widetilde{S}_{X}$ couplings. This stems from the fact that purely scalar interactions are not included in eq. (A.1).

The equivalence for the case of a pure pseudoscalar can be explicitly illustrated by comparing the analytical expressions obtained with eqs. (2.1) and (A.1) for a given observable. We can start with a trivial example, the process $\ell_{\alpha} \rightarrow \ell_{\beta} \phi$, discussed in section 4.1. Using the parametrization in eq. (A.1), one can easily derive the decay width of this two-body decay,

$$
\widetilde{\Gamma}\left(\ell_{\alpha} \rightarrow \ell_{\beta} \phi\right)=\frac{m_{\alpha}^{3}}{32 \pi}\left(\left|\widetilde{S}_{L}^{\beta \alpha}\right|^{2}+\left|\widetilde{S}_{R}^{\beta \alpha}\right|^{2}\right)
$$

where terms proportional to $m_{\beta}$ have been neglected. This results differs from eq. (4.1) only by a factor $m_{\alpha}^{2}$, as one would obtain from the direct application of the dictionary in eqs. (A.4) and (A.5). Let us now consider a less trivial example: $\ell_{\alpha}^{-} \rightarrow \ell_{\beta}^{-} \ell_{\beta}^{+} \ell_{\beta}^{-}$. The computation of its amplitude with the Lagrangian in eq. (A.1) makes use of the same Feynman diagrams shown in figure 3 . In this case one obtains

$$
\begin{aligned}
\widetilde{\mathcal{M}}_{\phi}= & \bar{u}\left(p_{3}\right) 2(-\not 1)\left(\widetilde{S}_{L}^{\beta \beta} P_{L}+\widetilde{S}_{R}^{\beta \beta} P_{R}\right) v\left(p_{4}\right) \frac{i}{q^{2}+i \varepsilon} \bar{u}\left(p_{2}\right)(\not l)\left(\widetilde{S}_{L}^{\beta \alpha} P_{L}+\widetilde{S}_{R}^{\beta \alpha} P_{R}\right) u\left(p_{1}\right) \\
& -\bar{u}\left(p_{2}\right) 2(-\not k)\left(\widetilde{S}_{L}^{\beta \beta} P_{L}+\widetilde{S}_{R}^{\beta \beta} P_{R}\right) v\left(p_{4}\right) \frac{i}{k^{2}+i \varepsilon} \bar{u}\left(p_{3}\right)(\not k)\left(\widetilde{S}_{L}^{\beta \alpha} P_{L}+\widetilde{S}_{R}^{\beta \alpha} P_{R}\right) u\left(p_{1}\right),
\end{aligned}
$$

where the factor of 2 preceding the diagonal coupling is due to the addition of the Hermitian conjugate, as explicitly shown in eq. (A.1). Again, explicit flavor indices have been 
introduced. The decay width is computed to be

$$
\begin{aligned}
& \widetilde{\Gamma}_{\phi}\left(\ell_{\alpha}^{-} \rightarrow \ell_{\beta}^{-} \ell_{\beta}^{+} \ell_{\beta}^{-}\right)= \\
& \quad \frac{m_{\alpha}^{5}}{512 \pi^{3}}\left\{4\left(\left|\widetilde{S}_{L}^{\beta \alpha}\right|^{2}+\left|\widetilde{S}_{R}^{\beta \alpha}\right|^{2}\right)\left(\widetilde{S}_{L}^{\beta \beta}-\widetilde{S}_{R}^{\beta \beta}\right)^{2} \frac{m_{\beta}^{2}}{m_{\alpha}^{2}}\left(4 \log \frac{m_{\alpha}}{m_{\beta}}-\frac{15}{2}\right)\right. \\
& +\frac{m_{\beta}}{3 m_{\alpha}}\left\{( \widetilde { S } _ { L } ^ { \beta \beta } - \widetilde { S } _ { R } ^ { \beta \beta } ) \left\{\widetilde{S}_{R}^{\beta \alpha}\left(A_{L L}^{S *}-2 A_{L R}^{S *}\right)-\widetilde{S}_{L}^{\beta \alpha}\left(A_{R R}^{S *}-2 A_{R L}^{S *}\right)\right.\right. \\
& +\frac{m_{\beta}}{m_{\alpha}}\left\{\widetilde{S}_{L}^{\beta \alpha}\left[2 A_{L L}^{S *}+\left(12 \log \frac{m_{\alpha}}{m_{\beta}}-25\right) A_{L R}^{S *}\right]-\widetilde{S}_{R}^{\beta \alpha}\left[2 A_{R R}^{S *}+\left(12 \log \frac{m_{\alpha}}{m_{\beta}}-25\right) A_{R L}^{S *}\right]\right\} \\
& +12\left[A_{R R}^{T *}\left(\widetilde{S}_{L}^{\beta \alpha}+2 \frac{m_{\beta}}{m_{\alpha}} \widetilde{S}_{R}^{\beta \alpha}\right)-A_{L L}^{T *}\left(\widetilde{S}_{R}^{\beta \alpha}+2 \frac{m_{\beta}}{m_{\alpha}} \widetilde{S}_{L}^{\beta \alpha}\right)\right]+4\left(\widetilde{S}_{R}^{\beta \alpha} A_{L R}^{V *}-\widetilde{S}_{L}^{\beta \alpha} A_{R L}^{V *}\right) \\
& +2 \frac{m_{\beta}}{m_{\alpha}}\left\{\widetilde{S}_{L}^{\beta \alpha}\left[\left(25-12 \log \frac{m_{\alpha}}{m_{\beta}}\right) A_{L R}^{V *}-\left(42-24 \log \frac{m_{\alpha}}{m_{\beta}}\right) A_{L L}^{V *}\right]\right. \\
& \left.-\widetilde{S}_{R}^{\beta \alpha}\left[\left(25-12 \log \frac{m_{\alpha}}{m_{\beta}}\right) A_{R L}^{V *}-\left(42-24 \log \frac{m_{\alpha}}{m_{\beta}}\right) A_{R R}^{V *}\right]\right\} \\
& +6 e^{2}\left[\left(K_{2}^{L}\right)^{\beta \alpha *} \widetilde{S}_{L}^{\beta \alpha}-\left(K_{2}^{R}\right)^{\beta \alpha *} \widetilde{S}_{R}^{\beta \alpha}\right] \\
& \left.\left.\left.+4 e^{2} \frac{m_{\beta}}{m_{\alpha}}\left(\frac{3}{2}+\pi^{2}+6 \log { }^{2} 2-6 \log ^{2} \frac{m_{\alpha}}{m_{\beta}}\right)\left[\left(K_{2}^{R}\right)^{\beta \alpha *} \widetilde{S}_{L}^{\beta \alpha}-\left(K_{2}^{L}\right)^{\beta \alpha *} \widetilde{S}_{R}^{\beta \alpha}\right]\right\}+ \text { c.c. }\right\}\right\}
\end{aligned}
$$

where in this expression $A_{X Y}^{I}=\left(A_{X Y}^{I}\right)^{\beta \beta \beta \alpha}$. We note that infrarred divergences also occur in interference terms at this order in $\frac{m_{\beta}}{m_{\alpha}}$. This explains the appearance of several $\log$ factors. The decay width in eq. (A.9) can be compared to a previous result in the literature. The authors of [29] drop all interference terms in their calculation, and then their result must be compared to the first line in eq. (A.9). One can easily relate the $\widetilde{S}_{L, R}$ coefficients to the ones in [29] as

$$
V_{\beta \alpha}^{e} \equiv-\frac{1}{2}\left(\widetilde{S}_{L}^{\beta \alpha}+\widetilde{S}_{R}^{\beta \alpha}\right), \quad A_{\beta \alpha}^{e} \equiv \frac{1}{2}\left(\widetilde{S}_{R}^{\beta \alpha}-\widetilde{S}_{L}^{\beta \alpha}\right)
$$

for the flavor violating terms, and

$$
A_{\beta \beta}^{e} \equiv\left(\widetilde{S}_{R}^{\beta \beta}-\widetilde{S}_{L}^{\beta \beta}\right)
$$

for the flavor conserving ones. With this translation, it is easy to check that both results agree up to a global factor of $1 / 2$.

In order to compare the $\ell_{\alpha}^{-} \rightarrow \ell_{\beta}^{-} \ell_{\beta}^{+} \ell_{\beta}^{-}$decay widths obtained with both parametrizations we need an expanded version of eq. (4.20) that includes terms up to $\mathcal{O}\left(\frac{m_{\beta}}{m_{\alpha}}\right)$. This 
is given by

$$
\begin{aligned}
\Gamma_{\phi} & \left(\ell_{\alpha}^{-} \rightarrow \ell_{\beta}^{-} \ell_{\beta}^{+} \ell_{\beta}^{-}\right)= \\
& \frac{m_{\alpha}}{512 \pi^{3}}\left\{\left(\left|S_{L}^{\beta \alpha}\right|^{2}+\left|S_{R}^{\beta \alpha}\right|^{2}\right)\left\{\left|S^{\beta \beta}\right|^{2}\left(4 \log \frac{m_{\alpha}}{m_{\beta}}-\frac{49}{6}\right)-\frac{2}{6}\left[\left(S^{\beta \beta *}\right)^{2}+\left(S^{\beta \beta}\right)^{2}\right]\right\}\right. \\
& -\frac{m_{\alpha}^{2}}{6}\left\{S_{L}^{\beta \alpha} S^{\beta \beta} A_{L L}^{S *}+2 S_{L}^{\beta \alpha} S^{\beta \beta *} A_{L R}^{S *}+2 S_{R}^{\beta \alpha} S^{\beta \beta} A_{R L}^{S *}+S_{R}^{\beta \alpha} S^{\beta \beta *} A_{R R}^{S *}\right. \\
& -12\left(S_{L}^{\beta \alpha} S^{\beta \beta} A_{L L}^{T *}+S_{R}^{\beta \alpha} S^{\beta \beta *} A_{R R}^{T *}\right)-4\left(S_{R}^{\beta \alpha} S^{\beta \beta} A_{R L}^{V *}+S_{L}^{\beta \alpha} S^{\beta \beta *} A_{L R}^{V *}\right) \\
& +6 e^{2}\left(S_{R}^{\beta \alpha} S^{\beta \beta} K_{2}^{L *}+S_{L}^{\beta \alpha} S^{\beta \beta *} K_{2}^{R *}\right)-\frac{36 m_{\beta}}{m_{\alpha}}\left(S_{R}^{\beta \alpha} S^{\beta \beta} A_{L L}^{T *}+S_{L}^{\beta \alpha} S^{\beta \beta *} A_{R R}^{T *}\right) \\
& +\frac{3 m_{\beta}}{2 m_{\alpha}}\left[S^{\beta \beta}\left(11 S_{L}^{\beta \alpha} A_{R L}^{S *}+2 S_{R}^{\beta \alpha} A_{L L}^{S *}-7 S_{R}^{\beta \alpha} A_{L R}^{S *}\right)\right. \\
& \left.+S^{\beta \beta *}\left(11 S_{R}^{\beta \alpha} A_{L R}^{S *}+2 S_{L}^{\beta \alpha} A_{R R}^{S *}-7 S_{L}^{\beta \alpha} A_{R L}^{S *}\right)\right] \\
& -\frac{6 m_{\beta}}{m_{\alpha}}\left(S_{L}^{\beta \alpha} A_{R L}^{S *}-S_{R}^{\beta \alpha} A_{L R}^{S *}\right)\left(S^{\beta \beta}-S^{\beta \beta *}\right) \log \frac{m_{\alpha}}{m_{\beta}} \\
& +\frac{12 m_{\beta}}{m_{\alpha}}\left(S_{L}^{\beta \alpha} A_{R L}^{V *}-2 S_{L}^{\beta \alpha} A_{R R}^{V *}+2 S_{R}^{\beta \alpha} A_{L L}^{V *}-S_{R}^{\beta \alpha} A_{L R}^{V *}\right)\left(S^{\beta \beta}-S^{\beta \beta *}\right) \log \frac{m_{\alpha}}{m_{\beta}} \\
& +\frac{3 m_{\beta}}{m_{\alpha}}\left[S^{\beta \beta}\left(-11 S_{L}^{\beta \alpha} A_{R L}^{V *}+14 S_{L}^{\beta \alpha} A_{R R}^{V *}-14 S_{R}^{\beta \alpha} A_{L L}^{V *}+7 S_{R}^{\beta \alpha} A_{L R}^{V *}\right)\right. \\
+ & \left.S^{\beta \beta *}\left(-11 S_{R}^{\beta \alpha} A_{L R}^{V *}+14 S_{R}^{\beta \alpha} A_{L L}^{V *}-14 S_{L}^{\beta \alpha} A_{R R}^{V *}+7 S_{L}^{\beta \alpha} A_{R L}^{V *}\right)\right] \\
& -4 e^{2} \frac{m_{\beta}}{m_{\alpha}}\left\{\left[S_{L}^{\beta \alpha}\left(K_{2}^{L}\right){ }^{\beta \alpha *}+S_{R}^{\beta \alpha}\left(K_{2}^{R}\right){ }^{\beta \alpha *}\right]\left(S^{\beta \beta}+S^{\beta \beta *}\right)\left(6 \log \frac{m_{\alpha}}{m_{\beta}}-\frac{21}{2}\right)\right. \\
+ & {\left.\left.\left.\left[S_{L}^{\beta \alpha} S^{\beta \beta}\left(K_{2}^{L}\right)^{\beta \alpha *}+S_{R}^{\beta \alpha} S^{\beta \beta *}\left(K_{2}^{R}\right)^{\beta \alpha *}\right]\left(\pi^{2}+6 \log { }^{2} 2-6 \log \frac{m_{\alpha}}{m_{\beta}}\right)\right\}+ \text { c.c. }\right\}\right\}, }
\end{aligned}
$$

where in this expression $A_{X Y}^{I}=\left(A_{X Y}^{I}\right)^{\beta \beta \beta \alpha}$. Replacing eqs. (A.4) and (A.5) into eq. (A.12) one finds full agreement with eq. (A.9) to order $\mathcal{O}\left(\frac{m_{\beta}}{m_{\alpha}}\right)$. This proves explicitly the equivalence between both parametrizations in the calculation of $\ell_{\alpha}^{-} \rightarrow \ell_{\beta}^{-} \ell_{\beta}^{+} \ell_{\beta}^{-}$mediated by a pure pseudoscalar. 
Open Access. This article is distributed under the terms of the Creative Commons Attribution License (CC-BY 4.0), which permits any use, distribution and reproduction in any medium, provided the original author(s) and source are credited.

\section{References}

[1] L. Calibbi and G. Signorelli, Charged Lepton Flavour Violation: An Experimental and Theoretical Introduction, Riv. Nuovo Cim. 41 (2018) 71 [arXiv:1709.00294] [INSPIRE].

[2] MEG II collaboration, The design of the MEG II experiment, Eur. Phys. J. C 78 (2018) 380 [arXiv: 1801.04688 ] [INSPIRE].

[3] MEG II and Mu3E collaborations, Towards a new generation of Charged Lepton Flavour Violation searches at the Paul Scherrer Institut: The MEG upgrade and the Muße experiment, EPJ Web Conf. 234 (2020) 01011 [INSPIRE].

[4] Mu3e collaboration, The Muзe Experiment, Nucl. Phys. B Proc. Suppl. 248-250 (2014) 35.

[5] T. Aushev et al., Physics at Super B Factory, arXiv:1002.5012 [InSPIRE].

[6] Belle-II collaboration, Prospects for $\tau$ Lepton Physics at Belle II, in 17th Conference on Flavor Physics and CP-violation, (2019) [arXiv:1906.08950] [INSPIRE].

[7] Muon $g-2$ collaboration, Muon $(g-2)$ Technical Design Report, arXiv:1501.06858 [INSPIRE].

[8] R.D. Peccei and H.R. Quinn, CP Conservation in the Presence of Instantons, Phys. Rev. Lett. 38 (1977) 1440 [INSPIRE].

[9] S. Weinberg, A New Light Boson?, Phys. Rev. Lett. 40 (1978) 223 [inSPIRE].

[10] F. Wilczek, Problem of Strong $P$ and T Invariance in the Presence of Instantons, Phys. Rev. Lett. 40 (1978) 279 [INSPIRE].

[11] L. Di Luzio, M. Giannotti, E. Nardi and L. Visinelli, The landscape of QCD axion models, Phys. Rept. 870 (2020) 1 [arXiv: 2003.01100] [InSPIRE].

[12] J. Preskill, M.B. Wise and F. Wilczek, Cosmology of the Invisible Axion, Phys. Lett. B 120 (1983) 127 [INSPIRE].

[13] L.F. Abbott and P. Sikivie, A Cosmological Bound on the Invisible Axion, Phys. Lett. B 120 (1983) 133 [INSPIRE].

[14] M. Dine and W. Fischler, The Not So Harmless Axion, Phys. Lett. B 120 (1983) 137 [INSPIRE].

[15] A. Davidson and K.C. Wali, Minimal flavor unification via multigenerational Peccei-Quinn symmetry, Phys. Rev. Lett. 48 (1982) 11 [INSPIRE].

[16] F. Wilczek, Axions and Family Symmetry Breaking, Phys. Rev. Lett. 49 (1982) 1549 [INSPIRE].

[17] L. Calibbi, F. Goertz, D. Redigolo, R. Ziegler and J. Zupan, Minimal axion model from flavor, Phys. Rev. D 95 (2017) 095009 [arXiv: 1612.08040] [INSPIRE].

[18] Y. Ema, K. Hamaguchi, T. Moroi and K. Nakayama, Flaxion: a minimal extension to solve puzzles in the standard model, JHEP 01 (2017) 096 [arXiv: 1612.05492] [INSPIRE]. 
[19] T. Alanne, S. Blasi and F. Goertz, Common source for scalars: Flavored axion-Higgs unification, Phys. Rev. D 99 (2019) 015028 [arXiv:1807.10156] [INSPIRE].

[20] S. Centelles Chuliá, C. Döring, W. Rodejohann and U.J. Saldaña-Salazar, Natural axion model from flavour, JHEP 09 (2020) 137 [arXiv: 2005.13541] [INSPIRE].

[21] M. Reig, J.W.F. Valle and F. Wilczek, $\mathrm{SO}(3)$ family symmetry and axions, Phys. Rev. D 98 (2018) 095008 [arXiv:1805.08048] [INSPIRE].

[22] Y. Chikashige, R.N. Mohapatra and R.D. Peccei, Are There Real Goldstone Bosons Associated with Broken Lepton Number?, Phys. Lett. B 98 (1981) 265 [INSPIRE].

[23] G.B. Gelmini and M. Roncadelli, Left-Handed Neutrino Mass Scale and Spontaneously Broken Lepton Number, Phys. Lett. B 99 (1981) 411 [INSPIRE].

[24] J. Schechter and J.W.F. Valle, Neutrino Decay and Spontaneous Violation of Lepton Number, Phys. Rev. D 25 (1982) 774 [INSPIRE].

[25] C.S. Aulakh and R.N. Mohapatra, Neutrino as the Supersymmetric Partner of the Majoron, Phys. Lett. B 119 (1982) 136 [INSPIRE].

[26] J. Heeck, Neutrino Lines from Majoron Dark Matter, PoS NuFact2017 (2017) 138 [INSPIRE].

[27] M. Reig, J.W.F. Valle and M. Yamada, Light majoron cold dark matter from topological defects and the formation of boson stars, JCAP 09 (2019) 029 [arXiv: 1905.01287] [INSPIRE].

[28] W. Hu, R. Barkana and A. Gruzinov, Cold and fuzzy dark matter, Phys. Rev. Lett. 85 (2000) 1158 [astro-ph/0003365] [INSPIRE].

[29] F. Björkeroth, E.J. Chun and S.F. King, Flavourful Axion Phenomenology, JHEP 08 (2018) 117 [arXiv: 1806.00660] [INSPIRE].

[30] F. Björkeroth, L. Di Luzio, F. Mescia and E. Nardi, U(1) flavour symmetries as Peccei-Quinn symmetries, JHEP 02 (2019) 133 [arXiv:1811.09637] [INSPIRE].

[31] M.B. Gavela, R. Houtz, P. Quilez, R. Del Rey and O. Sumensari, Flavor constraints on electroweak ALP couplings, Eur. Phys. J. C 79 (2019) 369 [arXiv:1901.02031] [INSPIRE].

[32] M. Bauer, M. Neubert, S. Renner, M. Schnubel and A. Thamm, Axionlike Particles, Lepton-Flavor Violation, and a New Explanation of $a_{\mu}$ and $a_{e}$, Phys. Rev. Lett. 124 (2020) 211803 [arXiv: 1908.00008] [INSPIRE].

[33] Q. Bonnefoy, E. Dudas and S. Pokorski, Chiral Froggatt-Nielsen models, gauge anomalies and flavourful axions, JHEP 01 (2020) 191 [arXiv: 1909.05336] [INSPIRE].

[34] C. Cornella, P. Paradisi and O. Sumensari, Hunting for ALPs with Lepton Flavor Violation, JHEP 01 (2020) 158 [arXiv: 1911.06279] [INSPIRE].

[35] J. Albrecht, E. Stamou, R. Ziegler and R. Zwicky, Probing flavoured Axions in the Tail of $B_{q} \rightarrow \mu^{+} \mu^{-}$, arXiv:1911.05018 [inSPIRE].

[36] J. Martin Camalich, M. Pospelov, P.N.H. Vuong, R. Ziegler and J. Zupan, Quark Flavor Phenomenology of the QCD Axion, Phys. Rev. D 102 (2020) 015023 [arXiv:2002.04623] [INSPIRE].

[37] M. Endo, S. Iguro and T. Kitahara, Probing e $\mu$ flavor-violating ALP at Belle II, JHEP 06 (2020) 040 [arXiv: 2002.05948] [INSPIRE]. 
[38] S. Iguro, Y. Omura and M. Takeuchi, Probing $\mu \tau$ flavor-violating solutions for the muon $g-2$ anomaly at Belle II, JHEP 09 (2020) 144 [arXiv:2002.12728] [INSPIRE].

[39] L. Calibbi, D. Redigolo, R. Ziegler and J. Zupan, Looking forward to Lepton-flavor-violating $A L P s$, arXiv: 2006.04795 [INSPIRE].

[40] J. Heeck and H.H. Patel, Majoron at two loops, Phys. Rev. D 100 (2019) 095015 [arXiv: 1909.02029] [INSPIRE].

[41] W. Porod, F. Staub and A. Vicente, A Flavor Kit for BSM models, Eur. Phys. J. C 74 (2014) 2992 [arXiv: 1405.1434] [INSPIRE].

[42] G. Raffelt and A. Weiss, Red giant bound on the axion-electron coupling revisited, Phys. Rev. D 51 (1995) 1495 [hep-ph/9410205] [inSPIRE].

[43] R. Bollig, W. DeRocco, P.W. Graham and H.-T. Janka, Muons in supernovae: implications for the axion-muon coupling, Phys. Rev. Lett. 125 (2020) 051104 [arXiv:2005.07141] [INSPIRE].

[44] D. Croon, G. Elor, R.K. Leane and S.D. McDermott, Supernova Muons: New Constraints on Z'Bosons, Axions and ALPs, JHEP 01 (2021) 107 [arXiv: 2006.13942] [inSPIRE].

[45] L. Di Luzio, M. Fedele, M. Giannotti, F. Mescia and E. Nardi, Solar axions cannot explain the XENON1T excess, Phys. Rev. Lett. 125 (2020) 131804 [arXiv:2006.12487] [InSPIRE].

[46] I.M. Bloch, A. Caputo, R. Essig, D. Redigolo, M. Sholapurkar and T. Volansky, Exploring new physics with $O(\mathrm{keV})$ electron recoils in direct detection experiments, JHEP 01 (2021) 178 [arXiv: 2006.14521] [INSPIRE].

[47] W. DeRocco, P.W. Graham and S. Rajendran, Exploring the robustness of stellar cooling constraints on light particles, Phys. Rev. D 102 (2020) 075015 [arXiv:2006.15112] [INSPIRE].

[48] E. Masso and J. Redondo, Compatibility of CAST search with axion-like interpretation of PVLAS results, Phys. Rev. Lett. 97 (2006) 151802 [hep-ph/0606163] [INSPIRE].

[49] XENON collaboration, Excess electronic recoil events in XENON1T, Phys. Rev. D 102 (2020) 072004 [arXiv:2006.09721] [INSPIRE].

[50] A. Djouadi, The Anatomy of electro-weak symmetry breaking. II. The Higgs bosons in the minimal supersymmetric model, Phys. Rept. 459 (2008) 1 [hep-ph/0503173] [INSPIRE].

[51] D. Carmi, A. Falkowski, E. Kuflik, T. Volansky and J. Zupan, Higgs After the Discovery: A Status Report, JHEP 10 (2012) 196 [arXiv:1207.1718] [inSPIRE].

[52] I. Antoniou, Constraints on scalar coupling to electromagnetism, Grav. Cosmol. 23 (2017) 171 [arXiv: 1508.00985] [INSPIRE].

[53] C. Burrage, A.-C. Davis and D.J. Shaw, Active Galactic Nuclei Shed Light on Axion-like-Particles, Phys. Rev. Lett. 102 (2009) 201101 [arXiv:0902.2320] [INSPIRE].

[54] R. Ballou et al., Latest Results of the OSQAR Photon Regeneration Experiment for Axion-Like Particle Search, in 10th Patras Workshop on Axions, WIMPs and WISPs, (2014) [DOI] [arXiv:1410.2566] [INSPIRE].

[55] M. Hirsch, A. Vicente, J. Meyer and W. Porod, Majoron emission in muon and tau decays revisited, Phys. Rev. D 79 (2009) 055023 [Erratum ibid. 79 (2009) 079901] [arXiv:0902.0525] [INSPIRE]. 
[56] A. Abada, M.E. Krauss, W. Porod, F. Staub, A. Vicente and C. Weiland, Lepton flavor violation in low-scale seesaw models: SUSY and non-SUSY contributions, JHEP 11 (2014) 048 [arXiv: 1408.0138] [INSPIRE].

[57] S.M. Barr and A. Zee, Electric Dipole Moment of the Electron and of the Neutron, Phys. Rev. Lett. 65 (1990) 21 [Erratum ibid. 65 (1990) 2920] [INSPIRE].

[58] H.H. Patel, Package-X: A Mathematica package for the analytic calculation of one-loop integrals, Comput. Phys. Commun. 197 (2015) 276 [arXiv:1503.01469] [INSPIRE].

[59] F.J. Botella, F. Cornet-Gomez and M. Nebot, Electron and muon $g-2$ anomalies in general flavour conserving two Higgs doublets models, Phys. Rev. D 102 (2020) 035023 [arXiv: 2006. 01934] [INSPIRE].

[60] C.-Y. Chen, H. Davoudiasl, W.J. Marciano and C. Zhang, Implications of a light "dark Higgs" solution to the $g_{\mu}-2$ discrepancy, Phys. Rev. D 93 (2016) 035006 [arXiv: 1511.04715] [INSPIRE].

[61] A. Jodidio et al., Search for Right-Handed Currents in Muon Decay, Phys. Rev. D 34 (1986) 1967 [Erratum ibid. 37 (1988) 237] [INSPIRE].

[62] TWIST collaboration, Search for two body muon decay signals, Phys. Rev. D 91 (2015) 052020 [arXiv: 1409.0638] [inSPIRE].

[63] Mu3E collaboration, The Rare and Forbidden: Testing Physics Beyond the Standard Model with Muße, SciPost Phys. Proc. 1 (2019) 052 [arXiv:1812.00741] [InSPIRE].

[64] A.-K. Perrevoort, Sensitivity Studies on New Physics in the Muße Experiment and Development of Firmware for the Front-End of the Mu3e Pixel Detector, Ph.D. Thesis, Ruprecht-Karls-Universität Heidelberg (2018) [INSPIRE].

[65] ARGUS collaboration, A Search for lepton flavor violating decays $\tau \rightarrow e \alpha, \tau \rightarrow \mu \alpha, Z$. Phys. C 68 (1995) 25 [INSPIRE].

[66] E. De La Cruz-Burelo, M. Hernandez-Villanueva and A. De Yta-Hernandez, New method for beyond the Standard Model invisible particle searches in tau lepton decays, Phys. Rev. D 102 (2020) 115001 [arXiv: 2007.08239] [INSPIRE].

[67] MEG collaboration, Final Results of the MEG Experiment, Nuovo Cim. C 39 (2017) 325 [arXiv: 1606.08168] [INSPIRE].

[68] R.D. Bolton et al., Search for the Decay $\mu^{+} \rightarrow e^{+} \gamma$, Phys. Rev. Lett. 56 (1986) 2461 [INSPIRE].

[69] J.T. Goldman et al., Light Boson Emission in the Decay of the $\mu^{+}$, Phys. Rev. D 36 (1987) 1543 [INSPIRE].

[70] R.D. Bolton et al., Search for Rare Muon Decays with the Crystal Box Detector, Phys. Rev. D 38 (1988) 2077 [INSPIRE].

[71] J. Heeck and W. Rodejohann, Lepton flavor violation with displaced vertices, Phys. Lett. B 776 (2018) 385 [arXiv: 1710.02062] [INSPIRE].

[72] A. de Gouvêa and P. Vogel, Lepton Flavor and Number Conservation, and Physics Beyond the Standard Model, Prog. Part. Nucl. Phys. 71 (2013) 75 [arXiv:1303.4097] [inSPIRE].

[73] Particle Data Group collaboration, Review of Particle Physics, Phys. Rev. D 98 (2018) 030001 [INSPIRE]. 
[74] T. Aoyama, M. Hayakawa, T. Kinoshita and M. Nio, Tenth-Order QED Contribution to the Electron $g-2$ and an Improved Value of the Fine Structure Constant, Phys. Rev. Lett. 109 (2012) 111807 [arXiv: 1205.5368] [INSPIRE].

[75] T. Aoyama, M. Hayakawa, T. Kinoshita and M. Nio, Complete Tenth-Order QED Contribution to the Muon g-2, Phys. Rev. Lett. 109 (2012) 111808 [arXiv:1205.5370] [INSPIRE].

[76] S. Laporta, High-precision calculation of the 4-loop contribution to the electron $g-2$ in QED, Phys. Lett. B $\mathbf{7 7 2}$ (2017) 232 [arXiv:1704.06996] [InSPIRE].

[77] T. Aoyama, T. Kinoshita and M. Nio, Revised and Improved Value of the QED Tenth-Order Electron Anomalous Magnetic Moment, Phys. Rev. D 97 (2018) 036001 [arXiv:1712.06060] [INSPIRE].

[78] Muon $g-2$ collaboration, Final Report of the Muon E821 Anomalous Magnetic Moment Measurement at BNL, Phys. Rev. D 73 (2006) 072003 [hep-ex/0602035] [INSPIRE].

[79] F. Jegerlehner and A. Nyffeler, The Muon g-2, Phys. Rept. 477 (2009) 1 [arXiv:0902.3360] [INSPIRE].

[80] RBC and UKQCD collaborations, Calculation of the hadronic vacuum polarization contribution to the muon anomalous magnetic moment, Phys. Rev. Lett. 121 (2018) 022003 [arXiv: 1801.07224] [INSPIRE].

[81] S. Borsányi et al., Leading hadronic contribution to the muon 2 magnetic moment from lattice QCD, arXiv:2002.12347 [INSPIRE].

[82] A. Crivellin, M. Hoferichter, C.A. Manzari and M. Montull, Hadronic Vacuum Polarization: $(g-2)_{\mu}$ versus Global Electroweak Fits, Phys. Rev. Lett. 125 (2020) 091801 [arXiv: 2003.04886] [INSPIRE].

[83] M. Lindner, M. Platscher and F.S. Queiroz, A Call for New Physics: The Muon Anomalous Magnetic Moment and Lepton Flavor Violation, Phys. Rept. 731 (2018) 1 [arXiv: 1610.06587] [INSPIRE].

[84] G.F. Giudice, P. Paradisi and M. Passera, Testing new physics with the electron g-2, JHEP 11 (2012) 113 [arXiv: 1208.6583] [INSPIRE].

[85] ACME collaboration, Improved limit on the electric dipole moment of the electron, Nature 562 (2018) 355 [INSPIRE].

[86] Muon (G-2) collaboration, An Improved Limit on the Muon Electric Dipole Moment, Phys. Rev. D 80 (2009) 052008 [arXiv:0811.1207] [INSPIRE].

[87] S. Gori, G. Perez and K. Tobioka, KOTO vs. NA62 Dark Scalar Searches, JHEP 08 (2020) 110 [arXiv: 2005. 05170] [INSPIRE].

[88] G. Arcadi et al., Tritium beta decay with additional emission of new light bosons, JHEP 01 (2019) 206 [arXiv: 1811.03530] [INSPIRE].

[89] Y. Uesaka, Model identification in $\mu^{-} \rightarrow e^{-}$conversion with invisible boson emission using muonic atoms, Phys. Rev. D 102 (2020) 095007 [arXiv: 2005.07894] [INSPIRE].

[90] D. Aristizabal Sierra, M. Tortola, J.W.F. Valle and A. Vicente, Leptogenesis with a dynamical seesaw scale, JCAP 07 (2014) 052 [arXiv: 1405.4706] [INSPIRE].

[91] M.G. Folgado and V. Sanz, On the interpretation of non-resonant phenomena at colliders, arXiv:2005.06492 [INSPIRE]. 